\title{
Towards low-energy-light-driven bistable photoswitches: ortho-fluoroaminoazobenzenes
}

\author{
Kim Kuntze $^{1} \cdot$ Jani Viljakka ${ }^{1} \cdot$ Evgenii Titov $^{2}\left(\mathbb{D} \cdot\right.$ Zafar Ahmed $^{1} \cdot$ Elina Kalenius $^{3} \cdot$ Peter Saalfrank $^{2} \cdot$ Arri Priimagi $^{1}(\mathbb{1})$
}

Received: 23 September 2021 / Accepted: 17 November 2021 / Published online: 10 December 2021

(c) The Author(s) 2021

\begin{abstract}
Thermally stable photoswitches that are driven with low-energy light are rare, yet crucial for extending the applicability of photoresponsive molecules and materials towards, e.g., living systems. Combined ortho-fluorination and -amination couples high visible light absorptivity of $o$-aminoazobenzenes with the extraordinary bistability of $o$-fluoroazobenzenes. Herein, we report a library of easily accessible $o$-aminofluoroazobenzenes and establish structure-property relationships regarding spectral qualities, visible light isomerization efficiency and thermal stability of the cis-isomer with respect to the degree of $o$-substitution and choice of amino substituent. We rationalize the experimental results with quantum chemical calculations, revealing the nature of low-lying excited states and providing insight into thermal isomerization. The synthesized azobenzenes absorb at up to $600 \mathrm{~nm}$ and their thermal cis-lifetimes range from milliseconds to months. The most unique example can be driven from trans to cis with any wavelength from UV up to $595 \mathrm{~nm}$, while still exhibiting a thermal cis-lifetime of 81 days.
\end{abstract}

\section{Graphical abstract}<smiles>COc1cc(F)c(N=Nc2ccc(OC)c(N3CCCCC3)c2)c(N2CCCC2)c1</smiles>

trans-to-cis: $405 \mathrm{~nm}$ cis-to-trans: $595 \mathrm{~nm}$ cis-lifetime: $72 \mathrm{~h}$

trans-to-cis: $595 \mathrm{~nm}$ cis-to-trans: cis-lifetime: $81 \mathrm{~d}$

Evgenii Titov

titov@uni-potsdam.de

Arri Priimagi

arri.priimagi@tuni.fi

1 Smart Photonic Materials, Faculty of Engineering and Natural Sciences, Tampere University, P.O. Box 541, 33101 Tampere, Finland

2 Theoretical Chemistry, Institute of Chemistry, University of Potsdam, Karl-Liebknecht-Straße 24-25, 14476 Potsdam, Germany

3 Department of Chemistry, University of Jyväskylä, P.O. Box 35, 40014 Jyväskylä, Finland

\section{Introduction}

Photochromic molecular switches such as azobenzenes [1], diarylethenes [2,3] and spiropyrans [4-6] pave the way towards next-generation pharmaceuticals [7-11], catalysts [12-14] and functional materials [15-18] that can be activated or controlled with a spatially and temporally precise external stimulus-light. Azobenzene derivatives, whose geometry, dipole moment and other physical properties change drastically upon cis-trans isomerization around the 
$\mathrm{N}=\mathrm{N}$ bond, are particularly attractive photoswitches due to their synthetic versatility, good fatigue resistance and high isomerization quantum yields [1]. The photochemical properties of azobenzene photoswitches can be tuned with simple structural modifications to meet the requirements of a given application. In particular, many applications benefit from switching with low-energy and low-intensity light as well as from high thermal stability of the metastable cis-isomer. These attributes are crucial for switches used in living systems [19] or memories [20] and often times advantageous for applications in solar thermal fuel systems [21, 22] and soft-robotic materials [23-25] as well: high-energy irradiation generally has a degrading effect on the switch and its surroundings $[26,27]$ and, on the other hand, constant illumination is not always possible.

The observed absorption of azobenzene in the UV-Visible range is a combination of two transitions, the symmetry-allowed $\pi-\pi^{*}$ and the symmetry-forbidden $n-\pi^{*}$. While many substitution patterns red-shift the $\pi-\pi^{*}$ band significantly [19, 28, 29], this is accompanied by a drastic drop in the thermal stability of the cis-isomer [30], rendering these azobenzenes unsuitable for applications in photobiology where constant irradiation is not possible and nearbistable systems are, therefore, required. ortho-Substitution with suitable moieties, in turn, decouples the $n-\pi^{*}$ absorption bands of the cis and trans isomers, red-shifting it for the trans-form while simultaneously prolonging the cislifetime [31, 32]. However, while ortho-methoxylated [33], fluorinated [31, 34-36] and chlorinated [37-39] compounds enable efficient switching between two essentially bistable forms with visible light, high irradiation intensities or long illumination periods (hours for ortho-chlorinated compounds [38, 39]) are required for efficient switching because of the low molar absorptivity of the $n-\pi^{*}$ band. This is a drawback particularly when the photoswitch is illuminated through tissue or other light-scattering or -absorbing material. ortho-Amination increases the molar absorptivity but concurrently decreases the cis-lifetime to the time scale of seconds [19]. However, as their advantage, amino-substituted azobenzenes are resistant to glutathione that reduces less electron-rich compounds and hampers their applicability in biological systems [40, 41]. In addition, compared to halogens, amino substituents such as piperazine and proline derivatives provide additional functionalization sites in the ortho positions, enabling molecular structures unattainable with ortho-fluorinated or chlorinated compounds. Thus, it would be highly desirable to combine the best qualities of both $o$-amino- and $o$-halosubstituents.

Recently, we have shown that combined ortho-fluorination and amination yields azobenzenes with high molar absorptivity at visible wavelengths and tunable $c i s$-lifetimes [42]. Starting from mono- or di-ortho-fluorinated precursors, we selectively substituted one or both of the fluorines with pyrrolidine via a high-yielding nucleophilic aromatic substitution reaction, producing azobenzenes with a relatively long (3 days) or extremely short ( $1 \mathrm{~s})$ cis-lifetime depending on the substitution pattern. However, the studied library of molecules did not include tetra-ortho-substituted azobenzenes that have widely shown the best isomerization efficiencies and $c i s$-lifetimes among different classes of ortho-substituted azobenzenes [31, 34]. The study also gave no information on how the choice of amine affects the photochemical properties of the ortho-functionalized azobenzenes. Furthermore, even though the molar absorptivity in the visible region was high, efficient trans-cis isomerization was only achieved at relatively short wavelengths $(365-405 \mathrm{~nm})$. Hence, there is a need for systematic studies to better understand and utilize this class of azobenzenes.

Herein, we present a library of 41 ortho-aminofluoroazobenzenes acquired by substituting one or more of the fluorine atoms in mono-, di- and tetra-ortho-fluorinated azobenzenes with different secondary amines. Through a systematic survey of the photochemical properties of each compound, we explore the effects of (i) degree of substitution and (ii) choice of amine, searching for the optimal molecular structures that can be isomerized efficiently with low-energy light while maintaining long cis-lifetimes. The observed structure-property relationships are further rationalized with density functional theory (DFT) and timedependent DFT (TD-DFT) calculations which provide electronic insight into low-lying excited states and shed light on thermal isomerization. Additionally, we show that commercial and inexpensive L-proline derivatives can be used to create azobenzenes with similar photoswitching properties as the pyrrolidine-substituted ones, while enhancing water solubility and providing easy synthetic access to ortho-linkage to, e.g., bioactive molecules or polymer networks.

\section{Results and discussion}

\subsection{Synthesis}

Starting from the ortho-mono- and difluorinated azobenzene precursors 1-3-F and utilizing previously reported synthetic procedures [42], we complemented the mono- and di-orthosubstituted series with piperidino- and dimethylamino-substituted azobenzenes (Scheme 1); pyrrolidino-substituted equivalents were already published [42]. The degree of substitution can be easily controlled for the difluorinated precursors $\mathbf{2 - F}$ and $\mathbf{3 - F}$, yielding series $\mathbf{2}-\mathbf{x}_{\mathbf{1}}$ and $\mathbf{2}-\mathbf{x}_{\mathbf{2}}$, and $\mathbf{3}-\mathbf{x}_{1}$ and $\mathbf{3 - x _ { 2 }}$, respectively, $\mathbf{x}$ standing for the amine and subscript for the number of fluorines that have been substituted. This is because the activation Gibbs free energy for the second substitution is significantly greater than for the first reaction due to electron-donating nature of the amino 
<smiles>COc1ccc(N=Nc2ccc(OC)cc2)cc1</smiles><smiles>[R]c1cc(OC)ccc1N=Nc1ccc(OC)cc1[R]</smiles><smiles>[R7]c1cc(OC)cc([R])c1N=Nc1ccc(OC)cc1</smiles><smiles>[R]c1cc(OC)cc([R])c1N=Nc1c([R])cc(OC)cc1[R]</smiles>

1-F: $R^{1}=F$

2-F: $R^{1}, R^{2}=F$

3-F: $R^{1}, R^{3}=F$

4-F: $R^{1}, R^{2}, R^{3}, R^{4}=F$

$1-x: R^{1}=x$

$2-x_{1}: R^{1}=x, R^{2}=F$

$3-x_{1}: R^{1}=x, R^{3}=F$ mono- \&

$2-x_{2}: R^{1}, R^{2}=x \quad$ disubs.

$3-x_{2}: R^{1}, R^{3}=x$

$4-x_{1}: R^{1}=x, R^{2}, R^{3}, R^{4}=F$

4- $x_{2 \text {-sym }}: R^{1}, R^{3}=x, R^{2}, R^{4}=F$

4-x-asym: $R^{1}, R^{2}=x, R^{3}, R^{4}=F$ tetra-

$4-x_{3}: R^{1}, R^{2}, R^{3}=x, R^{4}=F$

$4-x_{4}: R^{1}, R^{2}, R^{3}, R^{4}=x$

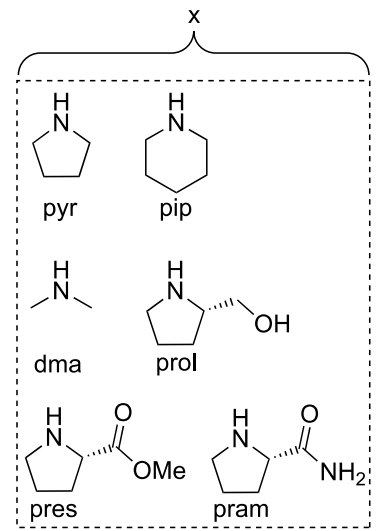

Scheme 1 Substitution patterns of studied azobenzene derivatives and the abbreviations used for different secondary amines. (pyr pyrrolidine, pip piperidine, dma dimethylamine, prol L-prolinol, pres L-proline methyl ester, pram L-prolinamide)

moiety, leading to excellent yields for either mono- or diaminated product [42]. The reaction proved to be less selective for the tetra-fluorinated 4-F, creating a mixture of $\mathbf{4}-\mathbf{x}_{\mathbf{1}}$, 4-x $\mathbf{x}_{2 \text {-sym }}$ and $\mathbf{4 - \mathbf { x } _ { 2 } \text { -asym }}$ (monoaminated, symmetrically diaminated and asymmetrically diaminated products, respectively) despite our efforts to optimize the reaction conditions. As an example, the optimized yields for 4-pip $\mathbf{p}_{1}$ and $\mathbf{4 - p i p _ { 2 - s y m }}$ only reached 58\% (85\% based on recovered starting material) and $54 \%$, respectively. The diaminated products 4 -pip -sym $_{\text {-sym }}$ and

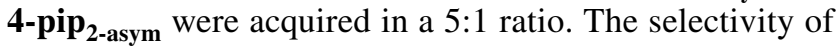
the pyrrolidine and piperidine substitution for 2-F, 3-F and 4-F is illustrated in Table 1. For the reactions of 4-F with other amines, the conditions were chosen so that all the products were acquired simultaneously.

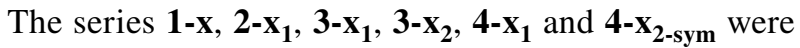
stable enough to be isolated and studied regardless of the chosen amine, but 2-pyr $\mathbf{2}_{\mathbf{2}}$ and 4-pyr $\mathbf{2}_{\mathbf{2} \text {-asym }}$ degraded over time. Of the three amines, piperidine produced the most stable products, demonstrated by the fact that the only stable products of higher degree of substitution were 4-pip ${ }_{3}$ and $\mathbf{4 p i p}_{\mathbf{4}}$; for pyrrolidine and dimethylamine, no stable products were acquired when more than two fluorines were substituted. Similar decomposition has been reported in earlier studies of ortho-amination [40, 42]. Conversely, the reaction times were longer for piperidine than for pyrrolidine and/or the former required heating in order to progress (Table 1),
Table 1 Selectivity of the amination of $\mathbf{2 - F}, \mathbf{3}-\mathbf{F}$ and $\mathbf{4 - F}$, the reaction scheme of entry 2 as an example

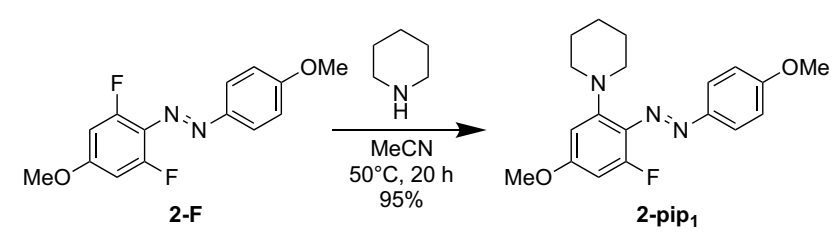

\begin{tabular}{|c|c|c|}
\hline Substrate & Reaction conditions & Product(s) and yield(s) \\
\hline $2-F+p^{a} r^{a}$ & 5 eq. in $\mathrm{MeCN}, \mathrm{rt}, 2.5 \mathrm{~h}$ & 2-pyr 1 (92\%) \\
\hline 2-F + pip & $\begin{array}{l}2 \text { eq. in } \mathrm{MeCN}, 50^{\circ} \mathrm{C} \text {, } \\
20 \mathrm{~h}\end{array}$ & 2-pip $1(95 \%)$ \\
\hline $3-F+p^{2} r^{a}$ & neat, rt, $20 \mathrm{~h}$ & 3-pyr 1 (81\%), 3-pyr 2 (15\%) \\
\hline 3-F + pip & 20 eq. in $\mathrm{MeCN}, \mathrm{rt}, 20 \mathrm{~h}$ & 3-pip $1(94 \%)$ \\
\hline 4-F + pyr & 2 eq. in $\mathrm{MeCN}, \mathrm{rt}, 2 \mathrm{~h}$ & $\begin{array}{l}\text { 4-pyr } \mathbf{p}_{1}(56 \%), \text { 4-pyr } \\
\quad(11 \%)\end{array}$ \\
\hline $4-F+p y r$ & 4 eq. in $\mathrm{MeCN}$, rt, $20 \mathrm{~h}$ & $\begin{array}{l}\text { 4-pyr } \mathbf{p}_{2-\mathbf{s y m}}(28 \%), 4-\mathbf{p y r}_{1} \\
\quad(10 \%)\end{array}$ \\
\hline 4-F + pip & 2 eq. in $\mathrm{MeCN}, 50^{\circ} \mathrm{C}, 2 \mathrm{~h}$ & $\begin{array}{l}\text { 4-pip } \\
\quad(58 \%), \text { 4-pip } \\
\quad(9 \%), \text { recovered 4-F } \\
(25 \%)\end{array}$ \\
\hline 4-F + pip & $\begin{array}{l}4 \text { eq. in } \mathrm{MeCN}, 50^{\circ} \mathrm{C} \text {, } \\
20 \mathrm{~h}\end{array}$ & $\begin{array}{l}\text { 4-pip } 2 \text {-sym }(54 \%), \text { 4-pip } \\
\quad(11 \%), \text { ppip }_{2 \text {-asym }}(11 \%) \\
\text { 4pip }_{3}(6.7 \%)\end{array}$ \\
\hline
\end{tabular}

$r t$ room temperature

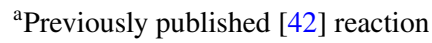

probably due to greater steric interactions in the transition state of the piperidine substitution.

Next, we investigated L-prolinol (prol), L-proline methyl ester (pres), and L-prolinamide (pram), using these pyrrolidine-resembling molecules to elaborate the series $\mathbf{2}-\mathbf{x}_{\mathbf{1}}, \mathbf{3}-\mathbf{x}_{\mathbf{1}}$, 4- $\mathbf{x}_{1}$ and $4-x_{2-s y m}$. The proline derivatives were consistently less reactive towards the fluorinated precursor than pyrrolidine (see Supporting Information), probably due to increased steric interactions and decreased nucleophilicity of the nitrogen lone pair. We also attempted to synthesize the L-prolinesubstituted products from $\mathbf{2 - F}$ to $\mathbf{4 - F}$ with the parent amino acid, but these could not be isolated, presumably because of intramolecular protonation of the azo bridge and subsequent decomposition. DFT calculations verify that the carboxylic proton is perfectly poised towards one of the nitrogen lone pairs at a hydrogen bonding distance (Fig. S142).

\subsection{Spectral properties}

Overall, 41 synthesized compounds were stable enough to be studied. The absorption wavelengths and molar extinction coefficients of the trans-isomer, cis-fractions in the photostationary state (PSS) and thermal lifetimes of the cis-to-trans isomerization of the compounds are presented in Table 2. The ortho-fluorinated azobenzenes 1-3-F exhibit typical 
Table 2 Photochemical properties and thermal cis-to-trans isomerization lifetime of synthesized azobenzenes ${ }^{\mathrm{a}}$

\begin{tabular}{|c|c|c|c|c|c|c|c|c|c|}
\hline & $\lambda(\mathrm{nm})$ & $\varepsilon\left(1 \mathrm{~mol}^{-1} \mathrm{~cm}^{-1}\right)$ & $\operatorname{PSS}(\% Z)^{\mathrm{b}}$ & $\tau_{c i s}{ }^{\mathrm{c}}$ & & $\lambda(\mathrm{nm})$ & $\varepsilon\left(1 \mathrm{~mol}^{-1} \mathrm{~cm}^{-1}\right)$ & $\operatorname{PSS}(\% Z)^{\mathrm{b}}$ & $\tau_{c i s}{ }^{\mathrm{c}}$ \\
\hline $1-F^{f}$ & $357 / 432$ & $29,110 / 3608$ & $93[365]$ & $42 \mathrm{~h}$ & 4-F & $343 / 435$ & $27,815 / 3086$ & $91[365]$ & $204^{\mathrm{d}}$ \\
\hline $2-F^{f}$ & $350 / 432$ & $24,652 / 2286$ & 93 [365] & $60 \mathrm{~h}$ & 4-pyr ${ }_{1}$ & $332 / 419 / 463$ & $10,686 / 4840 / 4956$ & $60[365]$ & $244 \mathrm{~h}$ \\
\hline $3-F^{f}$ & $343 / 435$ & $27,815 / 3086$ & $94[365]$ & $430 \mathrm{~h}$ & 4-pip 1 & $334 / 394 / 457$ & $8503 / 3975 / 2340$ & $71[365]$ & $81^{\mathrm{d}}$ \\
\hline $1-p y r^{f}$ & $340 / 430 / 462$ & $12,693 / 10818 / 12749$ & $72[470]$ & $15 \mathrm{~s}$ & 4-dma & $332 / 395 / 457$ & $15,219 / 6778 / 5064$ & $64[365]$ & $257 \mathrm{~h}$ \\
\hline 1-pip ${ }^{f}$ & $341 / 402 / 449$ & $14,924 / 10075 / 6800$ & 75 [405] & $9.1 \mathrm{~h}$ & 4-prol ${ }_{1}$ & $331 / 393 / 457$ & $5184 / 2824 / 2011$ & 39 [405] & $276 \mathrm{~h}$ \\
\hline 1-dma & $341 / 415 / 449$ & $11,421 / 7730 / 6943$ & $75[405]$ & $219 \mathrm{~s}$ & 4-pres 1 & $329 / 400 / 465$ & $15,818 / 6722 / 7227$ & 58 [405] & $260 \mathrm{~h}$ \\
\hline $2-$ pyr $_{1}{ }^{f}$ & $335 / 411 / 454$ & $16,739 / 8006 / 7792$ & $80^{\mathrm{d}}[405]$ & $72 \mathrm{~h}$ & 4-pram 1 & $329 / 394 / 466$ & $11,525 / 4717 / 4326$ & 55 [405] & $270 \mathrm{~h}$ \\
\hline 2-pip ${ }_{1}$ & $337 / 381 / 453$ & $15,930 / 8911 / 3493$ & $76[385]$ & $445 \mathrm{~h}$ & 4-pyr 2 -sym & $318 / 408 / 488$ & $7680 / 5661 / 5538$ & 54 [405] & $7.4 \mathrm{~h}$ \\
\hline 2-dma & $337 / 390 / 449$ & $17,179 / 8293 / 5555$ & $73[385]$ & $286 \mathrm{~h}$ & 4-pip 2-sym & $321 / 384 / 465$ & $5516 / 3060 / 2000$ & 43 [405] & $31 \mathrm{~h}$ \\
\hline 2-prol 1 & $333 / 405 / 456$ & $4955 / 2526 / 2307$ & 75 [385] & $131 \mathrm{~h}$ & 4-dma ${ }_{2 \text {-sym }}$ & $324 / 403 / 457$ & $13,103 / 9225 / 7178$ & $56[385]$ & $31 \mathrm{~h}$ \\
\hline 2-pres 1 & $339 / 396 / 447$ & $14,010 / 8144 / 7368$ & 62 [405] & $8.5 \mathrm{~h}$ & 4-prol 2 -sym & $317 / 387 / 487$ & 6111 & $44[405]$ & $75 \mathrm{~h}$ \\
\hline 2-pram 1 & $338 / 400 / 437$ & $13,362 / 7322 / 6544$ & $69[385]$ & $6.9 \mathrm{~h}$ & 4-pres 2 -sym & $324 / 388 / 482$ & $5175 / 5274 / 4346$ & $47[405]$ & $24 \mathrm{~h}$ \\
\hline $3-p \mathbf{r}_{1}{ }^{f}$ & $344 / 479$ & $13,777 / 15042$ & $71[405]$ & $258 \mathrm{~s}$ & 4-pram 2 -sym & $325 / 392 / 482$ & $5778 / 4039 / 3406$ & 60 [405] & $29 \mathrm{~h}$ \\
\hline 3-pip ${ }_{1}$ & $349 / 411 / 447$ & $16,610 / 10890 / 9228$ & $71[365]$ & $5 \mathrm{~h}$ & 4-pyr ${ }_{2 \text {-asym }}$ & $365 / 445$ & $5466 / 6200$ & $45[405]$ & $1.1 \mathrm{~s}$ \\
\hline 3-dma & $346 / 436 / 456$ & $16,315 / 11589 / 11804$ & 69 [405] & $165 \mathrm{~s}$ & 4-pip 2-asym & $374 / ?^{g}$ & $19,726 / ?^{\mathrm{g}}$ & 64 [405] & $192 \mathrm{~s}$ \\
\hline 3-prol 1 & $344 / 446 / 468$ & $9404 / 7590 / 8571$ & $61[405]$ & $219 \mathrm{~s}$ & 4-dma ${ }_{2 \text {-asym }}$ & $388 / ?^{g}$ & $10,350 / ?^{g}$ & 39 [405] & $0.2 \mathrm{~s}$ \\
\hline 3-pres 1 & $354 / 436 / 464$ & $14,391 / 7205 / 8159$ & 64 [405] & $74 \mathrm{~s}$ & 4-pram 2 -asym & $360 / 448$ & $6872 / 5770$ & $40[405]$ & $0.05 \mathrm{~s}$ \\
\hline 3-pram 1 & $343 / 436 / 462$ & $7068 / 6323 / 6777$ & $67[405]$ & $64 \mathrm{~s}$ & 4-pip 3 & $366 / 460$ & {$[\mathrm{e}]$} & 28 [405] & $3.8 \mathrm{~s}$ \\
\hline 2-pip 2 & 380 & 13,480 & 69 [365] & $10 \min$ & 4-pip 4 & $356 / 471$ & -[e] & $5[405]$ & $0.2 \mathrm{~s}$ \\
\hline 2-dma & 389 & 10,330 & 55 [365] & $0.4 \mathrm{~s}$ & & & & & \\
\hline $3-$ pyr $_{2}{ }^{f}$ & $488 / 514$ & $3701 / 3456$ & 62 [365] & $1.2 \mathrm{~s}$ & & & & & \\
\hline 3-pip 2 & $336 / 434$ & $10,110 / 12160$ & 61 [365] & $59 \mathrm{~min}$ & & & & & \\
\hline
\end{tabular}

${ }^{a}$ Studied in $50-150 \mu \mathrm{M}$ acetonitrile solutions. Spectral properties belong to the trans isomer

${ }^{\mathrm{b}}$ Minimum values estimated from the difference in absorbance. Irradiation wavelength is specified in square brackets

${ }^{\mathrm{c}}$ Thermal lifetime of the cis-isomer at $25{ }^{\circ} \mathrm{C}$, determined as an inverse of the rate constant in an exponential fit

${ }^{\mathrm{d}}$ Measured with ${ }^{1} \mathrm{H}$ NMR

${ }^{\mathrm{e}}$ Poorly soluble, accurate concentration not known

${ }^{\mathrm{f}}$ Previously published [42] data

${ }^{\mathrm{g}}$ Hard to extract peak value

azobenzene absorption spectra, strong $\pi-\pi^{*}$ peaks at ca. $350 \mathrm{~nm}$ accompanied by weak $\mathrm{n}-\pi^{*}$ bands at ca. $430 \mathrm{~nm}$. When fluorines are substituted with amines, the absorption spectrum changes significantly: the molar absorptivity in the $\pi-\pi^{*}$ band is lowered by half, while the absorptivity in the visible wavelengths (the $n-\pi^{*}$ band, $>400 \mathrm{~nm}$ ) increases multifold and is shifted towards red.

To explain the enhanced visible range absorption, we optimized the geometries of selected compounds at the B3LYP [43, 44] + D3(BJ)[45]/6-31G*[46] level of theory, using the polarizable continuum model $[47,48]$ (PCM) to account for the solvation effects by acetonitrile (see sect 4.4). The ortho-fluorinated precursor $\mathbf{1 - F}$ is perfectly planar with orthogonal $\mathrm{n}$ and $\pi^{*}$ orbitals, which accounts for the almost negligible absorbance in the $n-\pi^{*}$ band (Table 2), only non-zero due to molecular vibrations coupled with the electronic transitions [49]. The fact that the absorbance for the ortho-aminated compounds is relatively high in the $n-\pi^{*}$ band (the nature of the electronic transitions is discussed later in this section) would suggest that the planarity is broken. Optimized geometries indeed show that the amino-substituted compounds are twisted compared to their planar parent precursors. The degree of twisting can be quantified with three dihedral angles (Table 3): $\omega_{\mathrm{CCNN}}, \omega_{\mathrm{CNNC}}$ and $\omega_{\mathrm{NNCC}}$. For symmetrical azobenzenes, the first and third angle have the same value, and for asymmetrical ones, we have assigned $\omega_{\mathrm{CCNN}}$ to the amino-substituted side. While $\mathbf{1 F}$ is completely planar, its aminated derivatives exhibit dihedral angles of up to ca. $30^{\circ}$ on the side of the aminated ring. This improves the overlap between the $n$ and $\pi^{*}$ orbitals and explains the increased absorbance. We note, however, that the CCNN values of Table 3 alone cannot explain changes in absorbance observed when going, e.g., from 1-x to $\mathbf{2}-\mathbf{x}_{1}$. Apart from an effect of further $\mathrm{CN}$ torsion out of the equilibrium (discussed below), there is an intrinsic electronic effect of fluorine (see Supporting Information, p. S69). The red shift can be attributed to donation of electron density from 
Table 3 Dihedral angles and $\mathrm{C}-\mathrm{N}$ bond length of selected azobenzenes, calculated at the B3LYP+D3(BJ)/6-31G*/PCM(ACN) level of theory. The dihedral angles of interest are shown on the example of 4-pip1

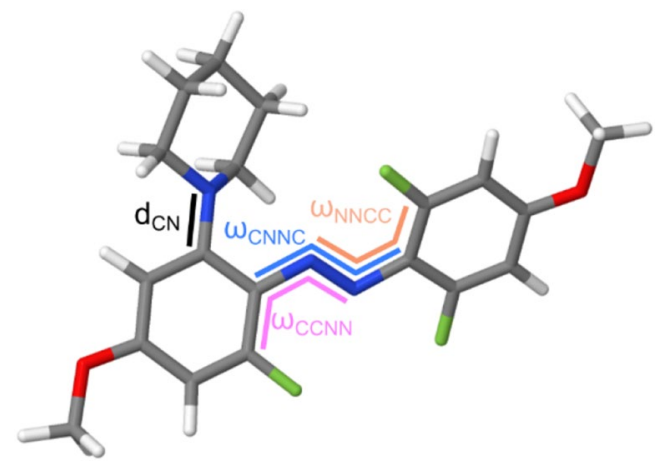

\begin{tabular}{|c|c|c|c|c|}
\hline & $\omega_{\mathrm{CCNN}}\left({ }^{\circ}\right)$ & $\omega_{\mathrm{CNNC}}\left({ }^{\circ}\right)$ & $\omega_{\mathrm{NNCC}}\left({ }^{\circ}\right)$ & $\mathrm{d}_{\mathrm{CN}}(\AA)$ \\
\hline 1-F & 0 & 180 & 0 & - \\
\hline 1-pyr & 2 & 180 & 3 & 1.368 \\
\hline 1-pip & 16 & 177 & 3 & 1.401 \\
\hline 2-pyr 1 & 23 & 177 & 8 & 1.369 \\
\hline 2-pip 1 & 29 & 177 & 4 & 1.398 \\
\hline 2-dma & 29 & 177 & 4 & 1.399 \\
\hline 3-pip 1 & 15 & 178 & 2 & 1.400 \\
\hline 4-pip & 28 & 177 & 7 & 1.394 \\
\hline 4-pip ${ }_{2-s y m}$ & 20 & 177 & 20 & 1.395 \\
\hline
\end{tabular}

the amino substituent, demonstrated by bond lengths of ca. 1.37-1.40 $\AA$ between the amino substituent and the aromatic ring, displaying significant double bond character (Table 3).

The effect that the substitution pattern has on the spectral properties is illustrated in Fig. $1 \mathrm{a}$ and $\mathrm{b}$ by comparing the spectra of different piperidino-substituted compounds. Piperidine was chosen because of the remarkable stability of the products, thus enabling the comparison of all relevant substitution patterns. Relative to ortho-fluoroazobenzenes, the absorptivity in the visible wavelength range increases the least for 2-pip $\mathbf{p}_{\mathbf{1}}$, i.e., when the fluorine and piperidine are located on the same aryl ring (red curve). Having only one piperidino substituent (1-pip, black curve) or a piperi-

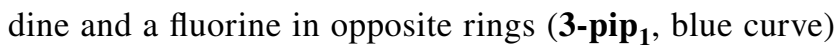
promotes visible light absorptivity more, with the absorp-

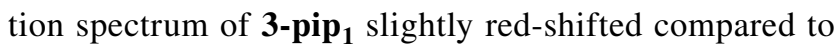
1-pip. Having two piperidines in the same ring (2-pip,

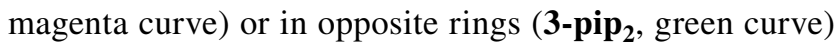
red-shifts the spectrum more pronouncedly, and the greatest visible range absorbance is exhibited by $3-$ pip $_{\mathbf{2}}$. The spectral shape is different for the asymmetrically diaminated molecules 2-pip $\mathbf{p}_{\mathbf{2}}$ and 4-pip $\mathbf{p}_{\mathbf{2}}$ asym compared to the symmetrical 3-pip - $_{2}$ and 4-pip 2-sym $_{\text {s. }}$ the former showing only one major absorption band in the visible range due to strong one-sided electron donation. The absorption maxima of the tetrasu-

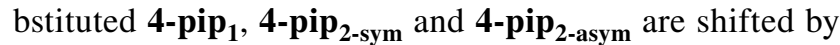
only a few nanometers compared to the respective disubstituted series, but the tail of the absorption band reaches significantly further on the red side, especially for 4-pip 2-sym $_{\text {-sym }}$ (Fig. 1b, red curve). Same trends were observed with other amines as well (Fig. S128-S138).

The choice of amine has a consistent effect on the absorption spectra: the compounds substituted with pyrrolidine or its derivatives show strong absorptivity from 400 to $550 \mathrm{~nm}$, whereas these peaks are smaller for piperidino- and dimethylamino-substituted molecules (Fig. 1c). The phenomenon is known for amino-substituted azobenzenes and arises from the degree of delocalization of the nitrogen lone pair, the nitrogen in the pyrrolidino moiety having more $\mathrm{sp}^{2}$ character than the ones in piperidino and dimethylamino substituents [50], as also demonstrated by shorter $\mathrm{C}-\mathrm{N}$ bond lengths for pyrrolidino-substituted compounds (Table 3 ). This difference is explained by steric interactions between the $\alpha$ protons of the amino group and the meta-proton in the aromatic ring that are stronger for piperidine and dimethylamine [50]. In addition, exo double bonds stabilize five-membered rings but destabilize six-membered rings [51]. Another trend is found in the photostationary state spectra: even though the compounds have almost equal cis-fractions upon illumination, the $n-\pi^{*}$ absorbance in the cis-rich mixture is highest for pyrrolidine, exceeding the absorbance of the pure trans isomer (and creating an isosbestic point) at the red end.

To further rationalize the experimental spectral data, we computed the excitation energies and oscillator strengths of the electronic transitions from the ground state to the low-lying excited states of selected molecules using linear response time-dependent density functional theory (TDDFT) [52] and PCM (in the non-equilibrium regime [53]) to account for solvent (ACN). We used B3LYP and $\omega$ B97X-D [54] density functional approximations as representatives of the global hybrid and long-range corrected families, respectively, as they are expected to give different results, in particular, not only different excitation energies but also ordering of excited states when intramolecular charge-transfer states are present $[55,56]$. The nature of excited states was analyzed by means of natural transition orbital [57] (NTO) analysis.

The structure-property relationships are reproduced on a semi-quantitative level computationally (see Fig. 2 for comparison of experimental and computational trends as well as Table S1 and Fig. S139 for calculated transitions and broadened absorption spectra, respectively). For the trans isomer, the amino substituents induce the splitting of the $\pi-\pi^{*}$ transition in two, one of which $\left(S_{0} \rightarrow S_{2}\right.$, the second band from the red side) is red-shifted and the other one $\left(S_{0} \rightarrow S_{3}\right.$, the third band) is blue-shifted with respect to 
(a)

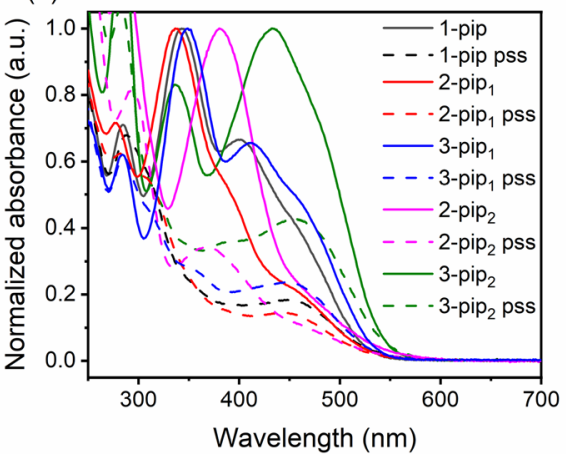

(b)

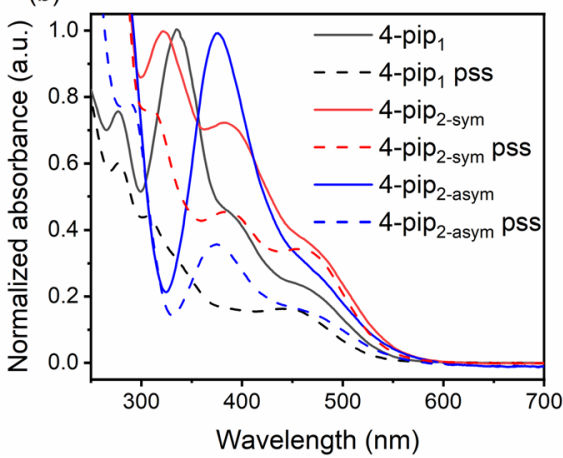

(c)

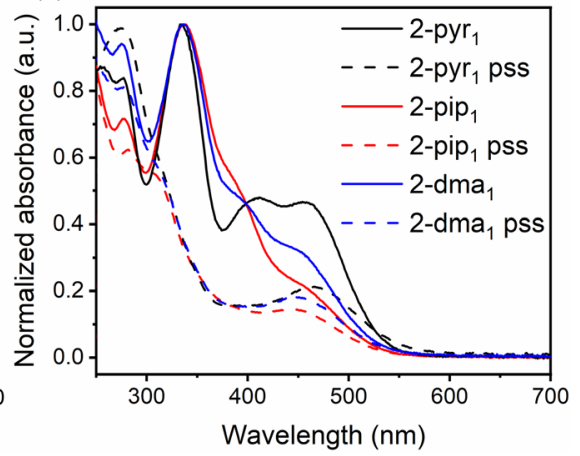

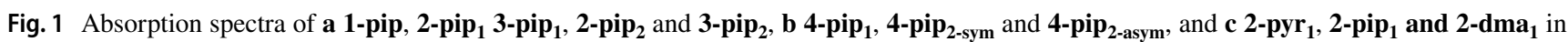
dark and in PSS, the most intense band normalized to 1

(a)

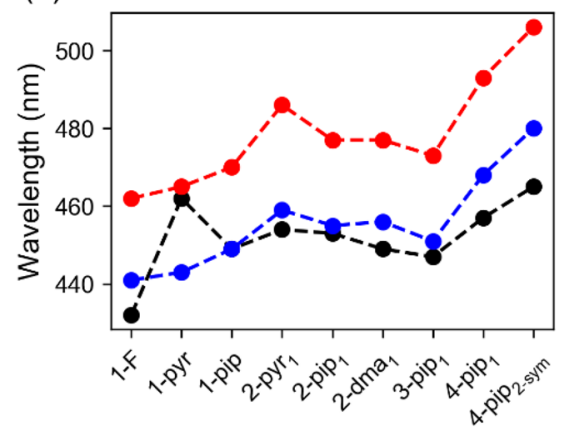

(d)

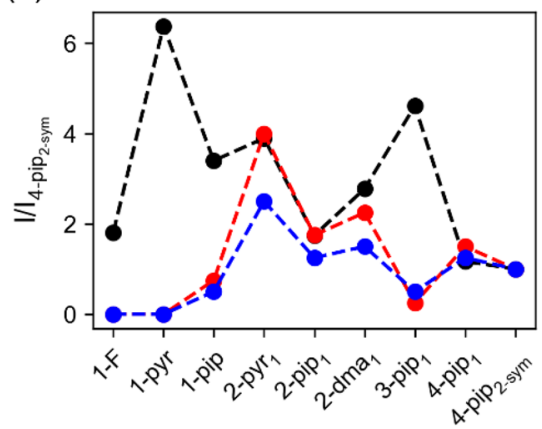

-๑- experiment $\quad-\bullet-B 3 L Y P \quad-\bullet-\omega B 97 X-D$

(b)

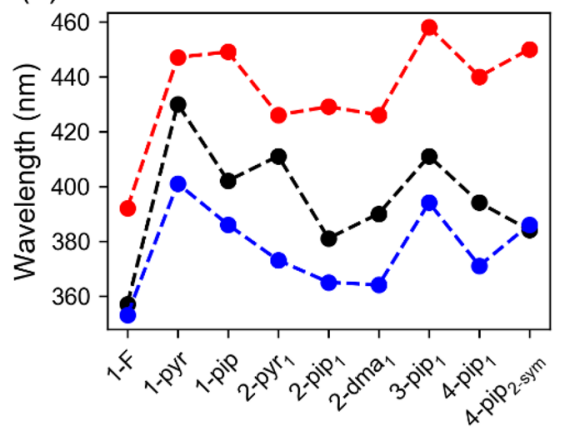

(e)

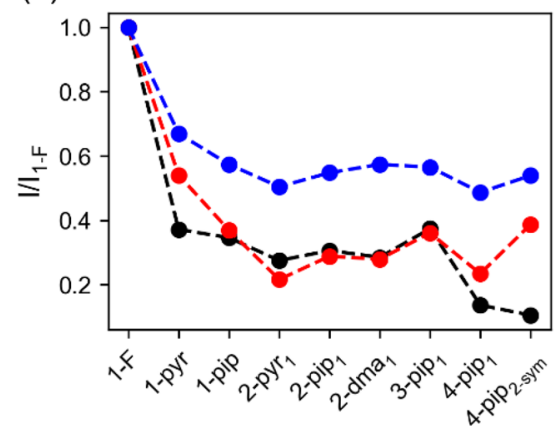

(c)

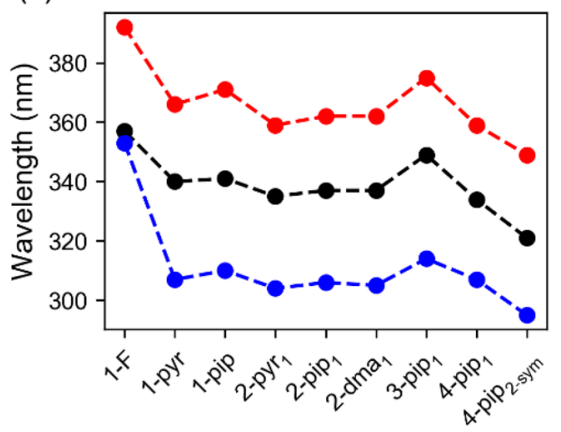

(f)

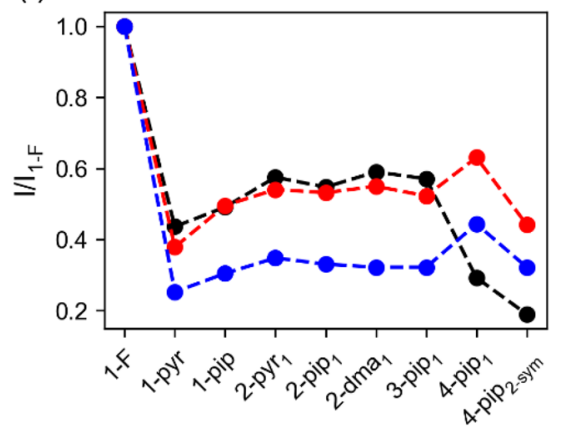

Fig. 2 Comparison of experimental and calculated peak positions $(\mathbf{a}-\mathbf{c})$ and relative intensities $(\mathbf{d}-\mathbf{f})$ for first three absorption bands of trans isomers (panels a,d for band 1; panels b,e for band 2; and panels $\mathrm{c}, \mathrm{f}$ for band 3 ). The relative intensity is calculated as the ratio

the $\pi-\pi^{*}$ transition $\left(S_{0} \rightarrow S_{2}\right)$ of the parent molecule 1-F. The hole NTOs of these transitions reveal considerable contribution of the nitrogen lone pair orbital of the amino substituent, especially for the second band (see Fig. 3 for the case of 2-pip ${ }_{1}$ and Table S2 for other molecules), giving the nitrogen substantial $\mathrm{sp}^{2}$ character. Moreover, the $\mathrm{n}-\pi^{*}$ transition $\left(\mathrm{S}_{0} \rightarrow \mathrm{S}_{1}\right)$ acquires a non-zero oscillator strength for all the considered aminoazobenzenes except of extinction coefficients (taken from Table 2) for the experimental trends and as the ratio of oscillator strengths (taken from Table S1) for the computational trends, relative to 4 -pip $\mathbf{p}_{2 \text {-sym }}$ in (d) and to $\mathbf{1 - F}$ in (e) and (f)

1-pyr. When using B3LYP, the $\mathrm{S}_{0} \rightarrow \mathrm{S}_{3}$ transition is usually more intense than the $S_{0} \rightarrow S_{2}$ transition. This trend is reversed when using $\omega \mathrm{B} 97 \mathrm{X}-\mathrm{D}$. The B3LYP trend appears to better reproduce experimental intensities, especially for the second and third bands (Fig. 2 e,f). We also note that B3LYP red-shifts the spectra with respect to the measured ones, whereas $\omega \mathrm{B} 97 \mathrm{X}-\mathrm{D}$ blue-shifts the $\pi-\pi^{*}$ transitions and either slightly red-shifts or blue-shifts the $n-\pi^{*}$ 


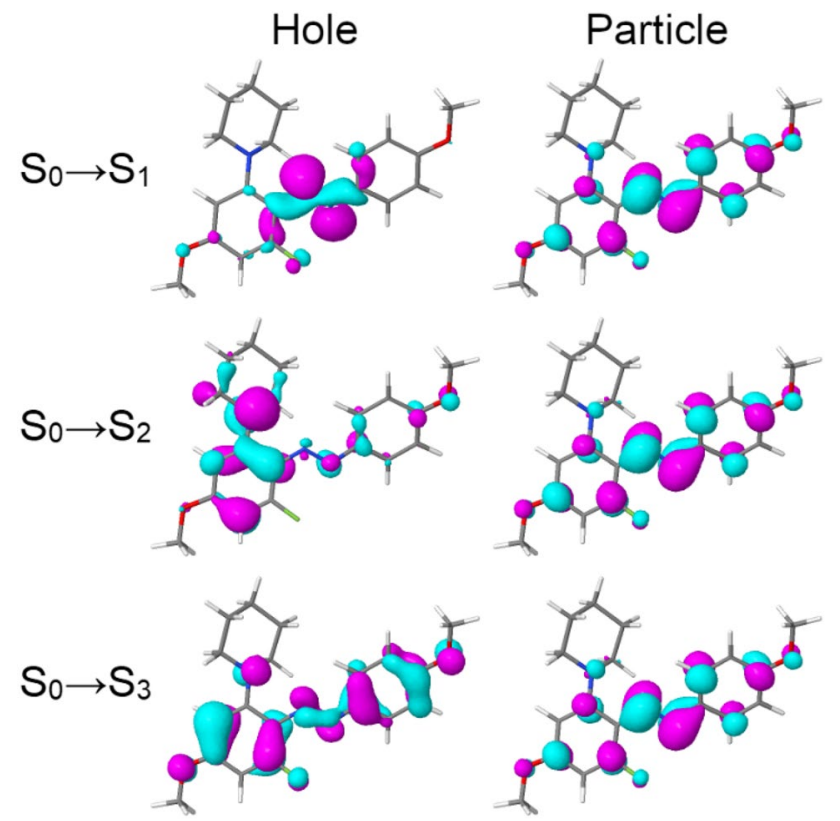

Fig. 3 Dominant natural transition orbital pairs for the first three transitions $\left(\mathrm{S}_{0} \rightarrow \mathrm{S}_{1}, \mathrm{~S}_{0} \rightarrow \mathrm{S}_{2}\right.$, and $\left.\mathrm{S}_{0} \rightarrow \mathrm{S}_{3}\right)$ of the trans-2-pip calculated at the TD-B3LYP + D3(BJ)/6-31G*/PCM(ACN) level of theory. Upon a given transition an electron is transferred from the "Hole" NTO to the "Particle" NTO. The contributions of the shown holeparticle pairs to the respective transitions are larger than $99 \%$

transition depending on the molecule in question (Fig. 2 $\mathrm{a}-\mathrm{c})$.

The $\mathrm{S}_{0} \rightarrow \mathrm{S}_{1}$ oscillator strengths of 2-pyr $\mathbf{r}_{1}$, 2-pip $\mathbf{p}_{\mathbf{1}}$ and 2-dma $\mathbf{a}_{\mathbf{1}}$ show the same trend as the experimental extinction coefficients (Fig. 2d). The $\mathrm{S}_{0} \rightarrow \mathrm{S}_{1}$ transition is also redshifted for 2-pyr $\mathbf{1}_{1}$ compared to the other two compounds. In addition, the computations reproduce the red shift 1-pi

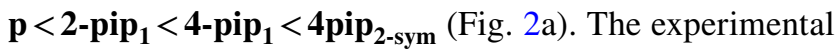
spectral differences in intensity between 2-pip ${ }_{1}$ and $\mathbf{3 - p i p _ { 1 }}$, however, are only reproduced computationally in the case of $S_{0} \rightarrow S_{2}$ and not for $S_{0} \rightarrow S_{1}$ (Fig. 2 d,e and Fig. S139). Explaining this would require computational studies beyond single geometries, e.g., molecular dynamics simulations or Wigner distributions [58]. Judging the performance of the two used functionals by comparing with the experiment, we find that TD- $\omega$ B97X-D performs particularly well for the excitation energies of the first band (Fig. 2a) and TDB3LYP works very well for the relative intensities of the second and third bands (Fig. 2 e,f). It is worth noting, however, that these results are obtained using an implicit solvation model (PCM in our case), which simplifies the complexity of the environment.

Interestingly, the $\mathrm{n}-\pi^{*}$ absorption band of 1-pyr is strong in the measured spectrum (Table 2), while zero $S_{0} \rightarrow S_{1}$ oscillator strength is found computationally (Table S1). To explain this discrepancy, we analyzed the change in the oscillator strength of the lowest transitions upon torsion around the $\mathrm{C}-\mathrm{N}$ bond (varying $\omega_{\mathrm{CCNN}}$ ), since this type of motion is expected to occur at room temperature. We found that this torsion does not require much energy and causes the increase in the $S_{0} \rightarrow S_{1}$ oscillator strength (Fig. S140), thus explaining the experimental results.

For the cis-isomer, we also observed the appearance of additional transitions in the low-energy region. Whereas for 1-F the $S_{0} \rightarrow S_{2}$ transition bears the largest oscillator strength (out of four lowest transitions), it is either the $S_{0} \rightarrow S_{3}$ or $\mathrm{S}_{0} \rightarrow \mathrm{S}_{4}$ transition which is the most intense one in the case of aminoazobenzenes, depending on the density functional in use and the molecule under consideration (Table S1). For most molecules, it is $\mathrm{S}_{0} \rightarrow \mathrm{S}_{4}$ with B3LYP and $\mathrm{S}_{0} \rightarrow \mathrm{S}_{3}$ with $\omega B 97 X-D$. The oscillator strengths of the $S_{0} \rightarrow S_{1}$ transition of $c i s-\mathbf{2}-\mathbf{p y r}_{\mathbf{1}}, c i s-\mathbf{2} \mathbf{p i p}_{\mathbf{1}}$ and $c i s-\mathbf{2}-\mathbf{d m a} \mathbf{a}_{\mathbf{1}}$ reproduce the experimentally observed trend in the absorbances of the cisrich photostationary state mixtures (pyr $>$ dma $>$ pip) with both functionals (Table S1 and Fig. S139). The NTOs of the cis-isomers are shown in Table $\mathrm{S} 3$.

\subsection{Photoisomerization efficiency}

To determine how efficiently the products can be switched from trans to cis, we estimated the cis-fraction in the most cis-rich PSS mixture by comparing the UV-Vis spectra in the PSS upon illumination $(365,385$ or $405 \mathrm{~nm})$ and in the dark after sufficient heating, i.e., with only trans-isomer present (see sect 4.2). The molar fractions should be viewed as minimum values, as the method assumes the cis-absorbance to be zero at the wavelength in question. As a representative example, the minimum cis-fraction in the PSS for 2-pyr $\mathbf{1}_{\mathbf{1}}$ upon irradiation with $405 \mathrm{~nm}$ light calculated with this method is $68 \%$, but with ${ }^{1} \mathrm{H}$ NMR measurements, $80 \%$ transto-cis switching is observed [42]. Similarly, upon $405 \mathrm{~nm}$ irradiation, the cis-fraction of 4-pip $_{1}$ was determined as $66 \%$ with ${ }^{1} \mathrm{H}$ NMR (Fig. S94c), compared to the $59 \%$ calculated from the UV-Vis spectra. The efficiency is best for the series 1-x, 2- $\mathbf{x}_{1}$ and 3-x $\mathbf{x}_{1}$, all of which can be driven to $>70 \%$ cis. The tetrasubstituted compounds cannot generally be isomerized as efficiently (Table 2).

We were particularly interested in switching with lowenergy illumination and screened excitation wavelengths from 365 to $595 \mathrm{~nm}$ for each product in the series 1-x, $\mathbf{2}-\mathbf{x}_{1}$, $\mathbf{3 - x _ { 1 }}, \mathbf{4 - x _ { 1 }}$ and $\mathbf{4} \mathbf{x}_{2 \text {-sym }}$, i.e., the series that are stable with all amino substituents. The cis-fractions upon illumination with specific wavelengths are presented in Table S5. It turns out that even though most compounds absorb strongly from 400 to $500 \mathrm{~nm}$, not all of them can be efficiently driven to the cis-state with $>435 \mathrm{~nm}$ illumination. For instance, the highly absorbing $\mathbf{2}-\mathbf{p y r}_{\mathbf{1}}$ is efficiently driven to cis with $365-435 \mathrm{~nm}$, but longer wavelengths drive the equilibrium towards trans (Fig. 4a). This holds true for many other 
(a)

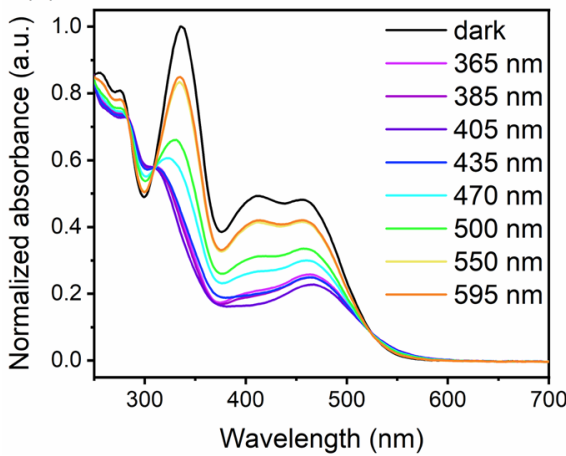

(b)

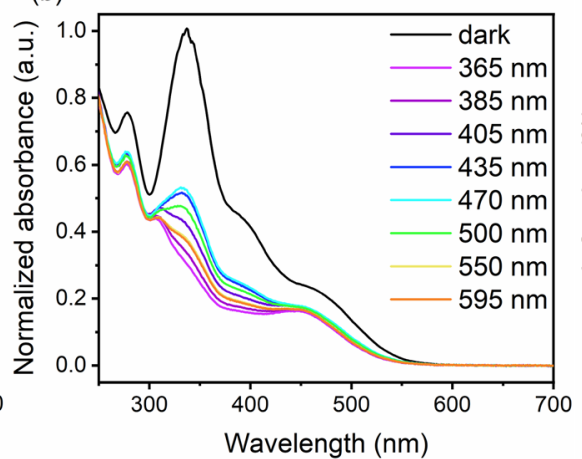

(c)

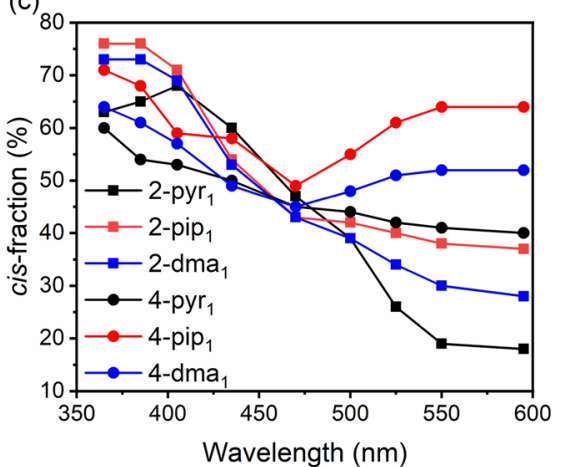

Fig. 4 Normalized absorption spectra of a 2-pyr $\mathbf{r}_{1}$ and $\mathbf{b}$ 4-pip $\mathbf{p}_{1}$ upon illumination with $365-595 \mathrm{~nm}$ light, and $\mathbf{c}$ the photostationary state compositions of selected azobenzenes with different illumination wavelengths

ortho-aminoazobenzenes as well, especially pyrrolidino- or proline-derivative-substituted ones that even exhibit an isosbestic point at ca. $520 \mathrm{~nm}$ (Fig. 1c, Fig. S134). Even though efficient isomerization is not possible at the absorbance maximum of the $\mathrm{n}-\pi^{*}$ region, this feature enables switching from trans to $c$ is with short wavelengths $(365-435 \mathrm{~nm})$ and in the opposite direction with lower energy light (525-595 nm). For piperidino- and dimethylamino-substituted compounds the back-isomerization is less effective (Fig. 4c), probably due to spectral differences for the cis-isomer (Fig. 1c). We wish to highlight that the photostationary states were acquired fast $(<30 \mathrm{~s})$ with illumination intensities ranging from 11 to $77 \mathrm{~mW} \mathrm{~cm}{ }^{-2}$ depending on the wavelength (Table S5).

The cis-to-trans photoisomerization is less efficient for the series $\mathbf{4 - x _ { 1 }}$ and $\mathbf{4}-\mathbf{x}_{\mathbf{2} \text {-sym }}$, hampering the switching between the isomers (Table 2). This is likely due to worse spectral separation of the isomers caused by increased twisting of the trans-isomer (Table 3). However, driving the equilibrium towards $c i s$ with low-energy light $(435-595 \mathrm{~nm})$ is more efficient than in the case of the disubstituted series. As the most promising example, 4-pip p $_{1}$ can be driven to $>64 \%$ cis state with 550 and $595 \mathrm{~nm}$ illumination (Fig. 4 b, c), a unique feature for thermally stable azobenzenes (cis-lifetime of 81 days at $25{ }^{\circ} \mathrm{C}$, see next section). In fact, with ${ }^{1} \mathrm{H}$ NMR studies, a cis-fraction of $72 \%$ was observed (Fig. S94d). Direct photoisomerization in the opposite direction is not effective, but the back-isomerization can be accomplished with, e.g., a red- or near-infrared-activated triplet sensitizer [59] or via electron transfer [60-63].

Considering the strong molar absorptivity of the pyrrolidine-substituted compounds in the visible range, it is interesting that the cis-to-trans isomerization is more efficient for piperidino- and dimethylamino-substituted azobenzenes at $400-500 \mathrm{~nm}$ (Fig. 4c). This is a good reminder that high molar absorptivity alone is not an indication of efficient switching if the spectral separation of the isomers and ratio of quantum yields are not favorable. The trend correlates with the absorbance of the cis-isomer at 400-600 nm which is highest for pyrrolidino- and lowest for piperidino-substituted azobenzenes (Fig. 1c, Fig. S139). As an interesting detail, the $c i$-fractions of 4-pyr $\mathbf{r}_{1}$ and $\mathbf{4 - p y r _ { 2 } \text { -sym }}$ decrease monotonically with increasing excitation wavelength, whereas for the piperidine and dimethylamine equivalents, the $c i s$-fraction decreases from 405 to $470 \mathrm{~nm}$ but increases when moving further to the red (Fig. 4c, Table S5).

\subsection{Thermal cis-trans isomerization rate}

The cis-lifetimes were determined by monitoring the absorbance values after trans-to-cis photoisomerization and fitting a monoexponential formula to the kinetic data. The lifetime $\tau$ is an inverse of the rate constant (see Sect 4.3). The thermal stability of the $c i s$-isomer is inversely correlated with the degree of $\pi$ donation from the amino group: when compounds with the same substitution pattern but different amino substituents are compared, the order in cis-lifetimes is piperidine $>$ dimethylamine $>$ pyrrolidine, reversed to the order seen for the molar absorptivity in the visible range. In effect, this means that the molecular design is often a trade-off between long cis-lifetimes and high absorptivity in the visible range, a typical dilemma of azobenzenes. In the series $\mathbf{1 - x}, \mathbf{3 - x _ { 1 }}, \mathbf{2}-\mathbf{x}_{\mathbf{2}}$ and $\mathbf{3 - \mathbf { x } _ { \mathbf { 2 } }}$, only piperidino products can be described as near-bistable, whereas the pyrrolidino- and dimethylamino-substituted azobenzenes isomerize in minutes or seconds. The series $\mathbf{2}-\mathbf{x}_{1}$, and 2-pyr $\mathbf{r}_{\mathbf{1}}$ in particular, provides a compromise: the cis-lifetimes range from 7 to $445 \mathrm{~h}\left(72 \mathrm{~h}\right.$ for $\left.2-\mathbf{p y r}_{1}\right)$, while the molar absorptivity remains high above $450 \mathrm{~nm}$.

In the earlier studies of ortho-fluorinated azobenzenes, tetra-ortho-fluorinated compounds exhibited the best cisstability [31]. When compared to series $\mathbf{2}-\mathbf{x}_{\mathbf{1}}$, the cis-lifetimes are indeed increased for series $\mathbf{4 - x _ { 1 }}$ (up to 81 days at $25{ }^{\circ} \mathrm{C}$ for 4-pip ${ }_{1}$ ) with the exception of $\mathbf{4 - d m a} \mathbf{d}_{1}$, for 
which the thermal isomerization is actually faster than for

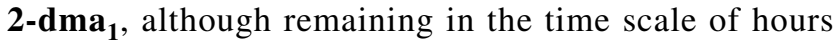
(Table 2). The stability of the cis-isomers in the series $\mathbf{4 - x _ { 2 } \text { -asym }}$ is even lower than in the series $\mathbf{2}-\mathbf{x}_{\mathbf{2}}$ (from 0.05 to $192 \mathrm{~s}$ ), probably due to a push-pull effect by the aminoand fluoro-substituents on opposite rings.

To corroborate our experimental findings, we studied the thermal isomerization kinetics computationally by Eyring's transition state theory [64] using the B3LYP functional which has been found to perform well for kinetics of azobenzene [65]. We considered both inversion and rotation pathways for the $c i s \rightarrow$ trans isomerization. Spinrestricted DFT (RDFT) calculations were done to locate inversion transition states and broken-symmetry spinunrestricted (UDFT) calculations were performed to find rotation transition states (we note that for 1-pyr we also found a rotation transition state using RDFT).

In the case of the inversion path, the linearization of the CNN unit may occur on either side from the azo group (Tables S10-S12). The intrinsic reaction coordinate (IRC) calculations $[66,67]$ with subsequent geometry optimizations showed that the cis-isomers corresponding to these two types of inversion transition states generally differ in geometry (conformation) from one another (Tables S10-S12). We note that these different cis-isomers of a given molecule have different ground-state energies (Table S6) but they may, in principle, be populated photochemically. See further discussion of different $c i s$-isomer conformations in Supporting Information (pp. S83-S85).

In the case of the RDFT treatment (inversion pathways), we performed calculations with and without solvent $(\mathrm{PCM}(\mathrm{ACN}))$ and dispersion $(\mathrm{D} 3(\mathrm{BJ}))$ corrections. The calculated lifetimes increase in the order gas phase $<\mathrm{PCM}(\mathrm{ACN})<\mathrm{PCM}(\mathrm{ACN})+\mathrm{D} 3(\mathrm{BJ})$, with the latter being too long in comparison with the experimental values (Table S6). The too long lifetimes can be attributed to too large calculated activation Gibbs free energies. Interestingly, the too large calculated inversion barriers (in comparison with experimental activation energies) when accounting for dispersion and solvation have recently been found for azobispyrazoles [68].

The lifetimes corresponding to the two inversion pathways are different, allowing one to assign a faster and a slower pathway (Fig. 5). That being said, the lifetime for the faster pathway (calculated taking into account PCM and $\mathrm{D} 3$ corrections) is still longer than the experimental lifetime.

For 1-pyr, we found an inversion and a rotation transition states (on the RDFT level) with virtually the same activation barrier. These two transition states correspond to the same cis conformation. In this case, the total rate constant was calculated as the sum of rate constants corresponding to rotation and inversion, and the lifetime was calculated as the

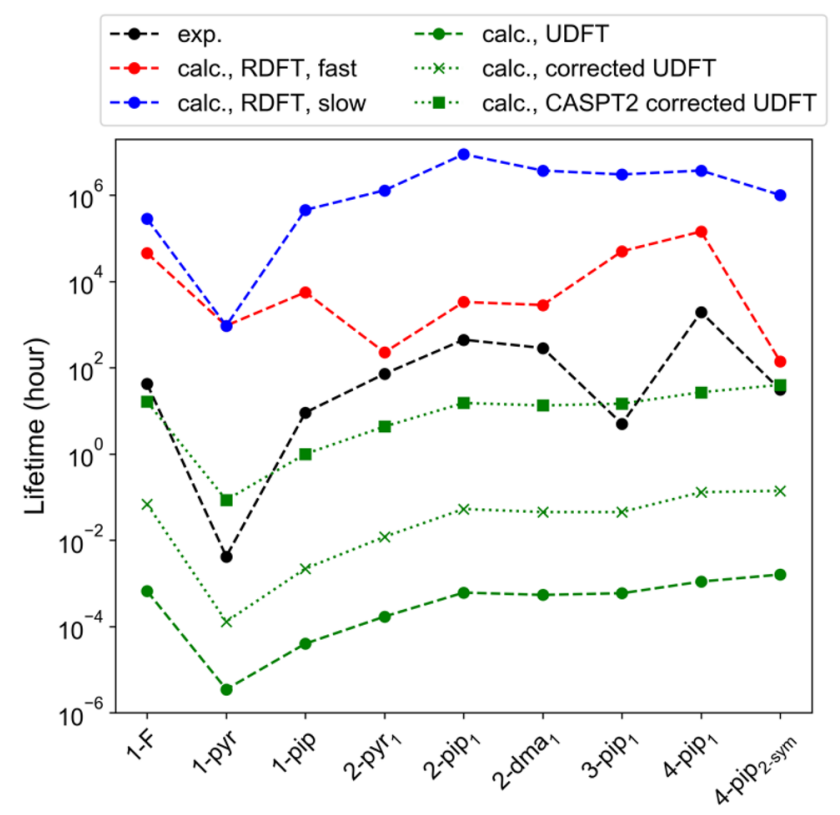

Fig. 5 Comparison of experimental and calculated lifetimes of ther$\mathrm{mal} \mathrm{Z} \rightarrow \mathrm{E}$ isomerization. The rotational pathway was treated with the $\mathrm{UB} 3 \mathrm{LYP}+\mathrm{D} 3(\mathrm{BJ}) / 6-31 \mathrm{G} / \mathrm{PCM}(\mathrm{ACN})$ method (green curves) and the inversion pathway with RB3LYP + D3(BJ)/6-31G*/PCM(ACN) (red and blue curves). "Corrected UDFT" corresponds to the activation barriers corrected using the approach by Yamaguchi et al. [69] "CASPT2 corrected UDFT" corresponds to $\Delta G_{U D F T}^{\ddagger}+0.26 \mathrm{eV}$ barriers, where $0.26 \mathrm{eV}$ comes from the difference in UDFT and CASPT2 energies of parent azobenzene (taken from ref [70]). See text for the details

inverse of the total rate constant. Yet, this lifetime is much larger than that found in experiment (Fig. 5).

We also considered the rotational pathway for all selected molecules applying broken-symmetry UDFT. The rotational transition states (with the CNNC dihedral of about $90^{\circ}$ and both CNN units bent) are expected to exhibit homolytic cleavage of the azo bond and, therefore, RDFT is expected to fail [70, 71]. Interestingly, for the recently introduced azobispyrazole family mentioned above, the rotational barriers calculated with UDFT were found to be lower than the experimental ones, in contrast to the inversion barriers (see above)[68].

We were able to locate rotation transition states for all selected molecules using the broken-symmetry UDFT, namely UB3LYP + D3(BJ)/6-31G*/PCM(ACN). The corresponding lifetimes calculated using UDFT barriers are too short (by a factor of $10^{-3}-10^{-6}$ ) when comparing to the experiment (Fig. 5). Yet, the $\left\langle S^{2}\right\rangle$ values (at the rotational TS geometries) are slightly above 1 for all the studied molecules, meaning that the electronic state is a mixed-spin state of singlet and higher multiplets. To estimate the energy of a pure-singlet state, with $\left\langle S^{2}\right\rangle=0$, (at the rotational TS geometry) we used the approach proposed by Yamaguchi et al. (see sect 4.4) [69]. The lifetimes calculated from the 
corrected barriers, while being shorter than the experimental ones, are closer to the experiment than the uncorrected UDFT lifetimes (Fig. 5). Moreover, for azobenzene, the difference between energies calculated with broken-symmetry UB3LYP and CASPT2 (at the rotational TS geometry) was found to be $\mathrm{E}_{\mathrm{CASPT} 2}-\mathrm{E}_{\mathrm{UB} 3 \mathrm{LYP}} \approx 0.26 \mathrm{eV}(6 \mathrm{kcal} /$ mol)[70]. Therefore, we also tried to correct $\Delta G_{U D F T}^{\ddagger}$ as $\Delta G_{U D F T}^{\ddagger}+0.26 \mathrm{eV}$, to estimate the correction coming from a multireference treatment. The lifetimes calculated using this approach (called "CASPT2 corrected UDFT") are in fairly good agreement with the experimental lifetimes. Moreover, the trend in experimental lifetimes with changing the amine is qualitatively reproduced (Fig. 5). In particular, for series $\mathbf{1 - x}$ and $\mathbf{2}-\mathbf{x}_{1}$ the experimental and computational trends coincide. We note that, while quantitative lifetimes may be wrong, the correct trends are also followed by the other theoretical treatments reported in Fig. 5.

Finally, several reports proposed that the thermal cis $\rightarrow$ trans isomerization may proceed via a nonadiabatic pathway involving the ground (lowest) singlet and triplet states, i.e. $\mathrm{S}_{0} \rightarrow \mathrm{T}_{1} \rightarrow \mathrm{S}_{0}$ transitions [70, 72, 73]. This hypothesis is based on (i) the fact that for azobenzene, the $\mathrm{T}_{1}$ state is located below the $\mathrm{S}_{0}$ state at the geometries close to the rotational transition state, and (ii) sufficiently large spin-orbit coupling along the rotational pathway [72].

To check the triplet energetics for our molecules, we calculated the energy of the $\mathrm{T}_{1}$ state at the rotational TS geometry. We also optimized the geometry in the $\mathrm{T}_{1}$ state (starting from the rotational transition state). Similar to the parent azobenzene, the triplet state $\left(\mathrm{T}_{1}\right)$ was found to be lower than the singlet state $\left(\mathrm{S}_{0}\right)$ at the geometries near the rotational transition state for all the molecules (Table S8). One thus cannot exclude the nonadiabatic pathway from consideration. We also note that the optimized triplet geometries are similar to the optimized UDFT rotational transition states, i.e. the minimum of the $\mathrm{T}_{1}$ PES is located on the rotational pathway.

In summary, our calculations indicate that for the investigated molecules, the thermal isomerization proceeds via rotation, which, in turn, may occur on the ground-state singlet PES or, probably, involves the nonadiabatic singlet-triplet pathway.

\subsection{Proline derivatives}

The spectral properties and photoswitching dynamics of the prolinol, proline ester and prolinamide series are close to those of the respective pyrrolidine-substituted azobenzenes: for example, 2-prol ${ }_{1}, \mathbf{2}$-pres ${ }_{1}$ and 2-pram 1 can all be cycled with 405 and $595 \mathrm{~nm}$ the same way as $\mathbf{2}-\mathbf{p y r}_{\mathbf{1}}$ (Table S5), and the cis-lifetime of 2-prol $\mathbf{1}_{1}$ is actually even longer than for 2-pyr $\mathbf{p}_{\mathbf{1}}$ (131 vs $72 \mathrm{~h}$, Table 2). Similarly, 3-pram $\mathbf{1}$ is isomerized to both directions even more efficiently than 3-pyr ${ }_{1}$. As an unexpected benefit, prolinamido-substituted products proved to be particularly robust towards decomposition over time, a problem especially associated with pyrrolidine-substituted azobenzenes.

In this light, the utilization of the proline derivatives for ortho-linkage to, e.g., biologically active molecules or polymeric materials while simultaneously gaining good photochemical properties seems feasible. We also noticed that the introduction of two prolinamido units into the system (4-pram 2sym $\left._{\text {sym }}\right)$ was enough to promote water solubility from 0 to $>500 \mu \mathrm{M}$ (Fig. S143) without compromising the photoswitching qualities. When more hydrophilic moieties are linked to the amines, even better water solubility will be attained.

\section{Conclusions}

We have synthesized and characterized 41 ortho-fluorinated and -aminated azobenzenes, varying the substitution pattern, degree of amination and choice of amine. Our simple synthetic routes yield ortho-aminofluoroazobenzenes, new photoswitches that combine the exceptionally long cis-lifetimes of ortho-fluorinated azobenzenes with the high visible-range absorptivity of ortho-aminated azobenzenes. Through systematic studies, we have determined how the aforementioned structural factors affect the photochemical properties. Compared to the parent ortho-fluoroazobenzenes, the degree to which the properties of the amino-substituted products differ depends on the $\pi$ donation strength of the amino substituent: a strong donor such as pyrrolidine promotes the visible light absorptivity more than other amines, whereas piperidine, a weaker donor, preserves the long cis-lifetime better. DFT calculations revealed non-planarity of the trans-isomers and TD-DFT calculations disclosed the nature of three absorption bands. The experimental trends for band shifts and changes in absorbance upon varying the substituents were semi-quantitatively reproduced. Moreover, the calculations indicated a preference for the rotational pathway for thermal cis $\rightarrow$ trans isomerization and corroborated trends within a given class of molecules.

Three of the studied substitution patterns (series $\mathbf{2}-\mathbf{x}_{1}$,

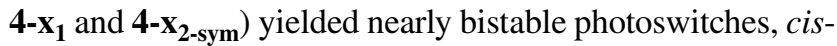
lifetimes in solution ranging from several hours to weeks at $25{ }^{\circ} \mathrm{C}$. Of these, the most promising switches were 2-pyr $\mathbf{r}_{1}$ that could be switched efficiently to both directions with 405 and $595 \mathrm{~nm}$ (trans-to-cis and cis-to-trans, respectively),

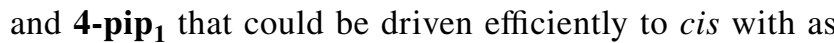
long wavelengths as $595 \mathrm{~nm}$. For applications where relatively fast (seconds to minutes) thermal isomerization is desired, series 1-x and 3-x $\mathbf{x}_{\mathbf{1}}$ provide systems that can be driven with visible light to either direction. In all cases, the 
photostationary state is reached extremely fast compared to, e.g., reported ortho-chlorinated azobenzenes. Lastly, we have shown that inexpensive L-proline derivatives can be used to synthesize switches with similar photochemical properties as the respective pyrrolidino-substituted compounds, paving the way towards easy ortho-linkage.

\section{Methods}

\subsection{Synthetic procedures}

Synthetic work was carried out in Tampere University. All reagents and solvents were commercial and purchased from Sigma Aldrich, TCI Europe, VWR or FluoroChem. The ortho-aminated azobenzenes were synthesized by stirring an ortho-fluorinated precursor and the selected amine in acetonitrile in ambient conditions. The amount of amine, reaction time and temperature were chosen with respect to the reactivity of the precursor and the desired degree of substitution (see Table 1 and Supplementary Methods). The synthe-

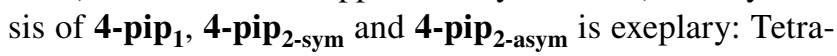
ortho-fluorinated precursor $\mathbf{4 - F}(31.1 \mathrm{mg}, 0.1 \mathrm{mmol})$ and pyrrolidine $(16.5 \mu \mathrm{l}, 0.2 \mathrm{mmol})$ were stirred in acetonitrile $(1 \mathrm{ml})$ at room temperature for $2 \mathrm{~h}$. The reaction mixture was then concentrated under reduced pressure and purified by column chromatography (10-20\% ethyl acetate in hexane) to yield 4-pyr $\mathbf{1}_{\mathbf{1}}$ (19 $\left.\mathrm{mg}, 52 \%\right)$ as an orange solid, 4-pyr $\mathbf{2}_{\text {-sym }}$ (4.0 $\mathrm{mg}, 9.6 \%)$ as a red solid and 4-pyr 2-asym $(4.0 \mathrm{mg}, 9.6 \%)$ as an orange-brown solid. Unreacted 4-F (5 mg, 16\%) was also recovered. Reactions were monitored with thin-layer chromatography (TLC) on commercial Merck Silica $60 \mathrm{~F}_{254}$ TLC plates, and the developed plates were visualized with UV irradiation $(254 \mathrm{~nm})$ or with potassium permanganate and cerium ammonium molybdate stains. The nuclear magnetic resonance (NMR) and high-resolution mass spectroscopy results are given in Supplementary Methods and NMR spectra are depicted in Fig. S1-S94.

Accurate mass experiments were performed with Agilent 6560 Ion mobility Q-TOF mass spectrometer, which was equipped with dualAJS ESI ion source. The compounds were dissolved in DCM $(1 \mathrm{mg} / \mathrm{ml})$ and samples were prepared with $1 \mu \mathrm{M}$ concentration in methanol. Samples were injected from syringe pump generally with $5 \mu \mathrm{l} / \mathrm{min}$ flowrate. ES tuning mix (Agilent technologies) was used for calibration. The exact mass values were calculated using Isotope Distribution Calculator (part of Agilent MassHunter Data Analysis Core) and the data was analyzed using MassHunter Workstation Software B08.00 from Agilent Technologies, USA. Characterization was done using accurate mass values (limit values $<3 \mathrm{mDa}$ or $<5 \mathrm{ppm}$ ) and fit of isotopic distributions. In case of overlapping distributions, the result was verified with measurement of ion mobility mass spectra (IM-MS).

The NMR spectra were measured with a $500 \mathrm{MHz}$ JEOL ECZR $500\left(125 \mathrm{MHz}\right.$ for $\left.{ }^{13} \mathrm{C}\right)$ at $25{ }^{\circ} \mathrm{C}$ and processed with the JEOL Delta NMR software version 5.3.1 (Windows).

\subsection{Photochemical studies}

UV-Visible absorption spectra were recorded with an Agilent Cary 60 spectrophotometer equipped with an Ocean Optics Qpod 2e Peltier-thermostated cell holder whose temperature accuracy is $0.1{ }^{\circ} \mathrm{C}$. Photoexcitation was conducted using a Prior Lumen 1600 light source containing multiple narrow-band LEDs at different wavelengths. The spectra of the LEDs were measured with the aforementioned spectrophotometer without taking into account the wavelength dependency in the sensitivity of the sensor. The illumination intensities were acquired by measuring the illumination powers with a Coherent LabMax thermal power meter and dividing the number by the area of the cuvette being illuminated $\left(0.78 \mathrm{~cm}^{2}\right)$. Full-width-halfmaximum values and illumination intensities are shown in Table S5. Quartz fluorescence cuvettes with an optical path of $1.0 \mathrm{~cm}$ were used for all measurements. The molar absorption coefficients were calculated with the Beer-Lambert law for each distinguishable absorption peak. For figures the absorption spectra were normalized to 1 with respect to the $\pi-\pi^{*}$ band.

Photostationary state (PSS) refers to the mixture of isomers upon constant illumination with a chosen excitation wavelength. Dark, in turn, refers to the mixture of isomers after an elongated period in a dark cavity at room temperature or at an elevated temperature. Based on the thermal relaxation lifetime of the molecules, the time and temperature were chosen so that the mixture would consist of azobenzenes only in the trans state.

The photostationary state compositions were determined by comparing the absorbance values of the photostationary state mixture with those in the dark spectrum. The cis-fraction was calculated from the wavelength that had the greatest difference in the absorbance values. If the cis-absorbance at this wavelength is assumed zero and the dark spectrum is assumed to equal to that of pure trans isomer, the cis-fraction is $1-A b s_{p s s} / A b s_{d a r k}$. This is a minimum value, as the cis-absorbance is typically nonzero. If the dark spectrum is not entirely trans (i.e., the mixture has not been in dark for long enough to isomerize completely to trans), this will also lower the calculated cis-fraction compared to the true value. However, these values are relatively accurate: for instance, for 4-dma $\mathbf{2}_{\text {-sym }}$ the isomer composition calculated from an NMR sample 
after prolonged irradiation is $58 \%$ cis and $42 \%$ trans (Fig. S94a), exactly the composition estimated from the UV-Vis spectra.

\subsection{Thermal isomerization studies}

The thermal isomerization rates were determined with the same methodology as in our earlier study [42]. The $50-150 \mu \mathrm{M}$ acetonitrile solutions were illuminated with 365 , 385 or $405 \mathrm{~nm}$ light until a photostationary state was reached. The illumination was then stopped and the absorbance near the absorption maximum of the trans isomer was monitored as a function of time. The measured absorbance values were fitted with a monoexponential function. If the thermal cis-totrans isomerization was fast, one measurement at $25^{\circ} \mathrm{C}$ was carried out. Otherwise, the rates were determined at 50, 60 and $70{ }^{\circ} \mathrm{C}$ and extrapolated to $25^{\circ} \mathrm{C}$ using the Arrhenius equation. The analyses are presented for all new compounds in Fig. S95-S127.

\subsection{Computational studies}

All calculations were done with Gaussian 16, revision C.01 [74]. The B3LYP functional [43, 44] with D3(BJ) [45] dispersion correction and $\mathrm{PCM}(\mathrm{ACN})[47,48]$ has been used for geometry optimizations to produce structures for spectra calculations. Low-lying electronically excited states have been calculated with TD-DFT [52] using B3LYP and $\omega$ B97X-D[54] functionals, with $\operatorname{PCM}(\mathrm{ACN})$. Natural transition orbitals (NTOs) [57] have been computed (using Gaussian) to visualize the nature of the excited states.

NTO analysis allows one to simplify an orbital description of electronic transitions. Often, a single NTO hole-particle pair may describe the transition in question whereas several conventional occupied-virtual orbital pairs are needed to characterize the same transition. Upon the electronic transition the excited electron goes from the hole NTO to the particle NTO.

The geometries of the transition states (TSs) and the cis $(\mathrm{Z})$ isomers have been optimized using spin-restricted $(\mathrm{R})$ und spin-unrestricted (U) B3LYP + D3(BJ)/6-31G*/PCM(ACN) calculations. In the case of spin-restricted calculations, we have also performed calculations without dispersion correction and without PCM to determine the influence of these corrections on thermal lifetimes. Reaction rate constants for the thermal cis $\rightarrow$ trans isomerization have been calculated using Eyring's transition state theory [64]:

$k=\frac{k_{B} T}{h} \exp \left(-\frac{\Delta G^{\ddagger}}{k_{B} T}\right)$

and the corresponding thermal lifetime as: $\tau=\frac{1}{k}$.

Here, $k_{B}$ and $h$ are the Boltzmann and Planck constants, respectively, and $T$ is the temperature. We used $T=298.15 \mathrm{~K}$ $\left(25^{\circ} \mathrm{C}\right) . \Delta G^{\ddagger}=G(\mathrm{TS})-G(\mathrm{Z})$ is the activation Gibbs free energy.

We located transition states using the Berny algorithm as implemented in Gaussian (TS keyword). Intrinsic reaction coordinate [66, 67] calculations (IRC keyword) have been done to locate the corresponding cis isomers, which have been further reoptimized. We have located two transition states and two cis isomers for each molecule. Energy differences between different $c i$ is isomers of the same molecule were calculated with respect to the lowest energy cis-isomer (of two isomers) of this molecule:

$\Delta B_{Z Z}^{r e l}=B\left(Z_{i}\right)-B\left(\mathrm{Z}_{\text {lowest energy }}\right) ; B=G, E ;$

$G$ is Gibbs free energy; $E$ is energy, i.e. a point on the potential energy surface (PES). Thus, $\Delta B_{Z Z}^{\text {rel }}=0$ for the lowest energy cis-isomer, and positive for the second isomer.

Normal mode analysis has been performed to confirm the nature of minima (no imaginary frequency) and transition states (one imaginary frequency).

In the case of spin-unrestricted calculations (performed at the UB3LYP + D3(BJ)/6-31G*/PCM(ACN) level), the Guess(Mix) keyword was used to generate an initial orbital guess, and the broken-symmetry solution was found for all considered molecules. The $\left\langle S^{2}\right\rangle$ values (at the rotational TS geometries) were slightly above 1 for all the molecules.

To estimate the energy of a pure-singlet state $\Delta E_{U D F T}^{(S, \ddagger)}$ (at the rotational TS geometry), we used an approach proposed by Yamaguchi et al. [69]

$\Delta E_{U D F T}^{(S, \ddagger)}=\Delta E_{U D F T}^{\ddagger}+\frac{\left\langle S^{2}\right\rangle}{2-\left\langle S^{2}\right\rangle}\left(\Delta E_{U D F T}^{\ddagger}-\Delta E_{U D F T}^{(T, \ddagger)}\right)$.

Here, the $\left\langle S^{2}\right\rangle$ values are for rotational TSs, i.e., about 1; $\Delta E_{U D F T}^{*}$ is the energy of the mixed-spin state, and $\Delta E_{U D F T}^{(T, \#)}$ is the energy of the triplet state at the rotational TS geometry. The second term on the r.h.s. was added to the UDFT activation Gibbs free energies $\Delta G_{U D F T}^{\ddagger}$ to obtain the corrected activation Gibbs free energies $\Delta G_{U D F T}^{(S, ;)}$ corresponding to the pure-singlet state.

The lowest triplet state was optimized starting from the rotational TS structures using the same level of theory, i.e., $\mathrm{UB} 3 \mathrm{LYP}+\mathrm{D} 3(\mathrm{BJ}) / 6-31 \mathrm{G}^{*} / \mathrm{PCM}(\mathrm{ACN})$. The $\left\langle S^{2}\right\rangle$ values were slightly above 2 , for the optimized triplets. The optimized triplet geometries are similar to the rotational TS structures.

In all the calculations, the $6-31 \mathrm{G}^{*}$ basis set [46] has been used. 
The molecular structures and NTOs have been visualized using Jmol, an open-source Java viewer for chemical structures in 3D (http://www.jmol.org/).

Supplementary Information The online version contains supplementary material available at https://doi.org/10.1007/s43630-021-00145-4.

Acknowledgements Financial support of the European Research Council (Starting Grant Project PHOTOTUNE, Decision Number 679646) is gratefully acknowledged. The work is conducted as part of the Academy of Finland Flagship Programme, Photonics Research and Innovation (PREIN), Decision Number 321065. K.K. is grateful for the financial support of Tampere University graduate school. E.T. is grateful to the Deutsche Forschungsgemeinschaft (DFG, German Research Foundation) for the financial support (project number 454020933). (Gefördert durch die Deutsche Forschungsgemeinschaft (DFG) - Projektnummer 454020933.) The authors would like to thank Prof. Stefan Hecht for many fruitful discussions on azobenzenes.

Author contributions $\mathrm{KK}$, JV and ZA carried out the experimental work, except for the mass spectra that were measured by EK, ET carried out the computational studies. KK and ET wrote the manuscript with the help of all authors. AP and PS initiated, supervised and funded the work, with partial funding acquired by KK and ET.

Funding H2020 European Research Council, 679646, Arri Priimägi, Luonnontieteiden ja Tekniikan Tutkimuksen Toimikunta, 321065, Graduate School, Tampere University, Deutsche Forschungsgemeinschaft, 454020933, Evgenii Titov

Data availability The datasets generated during and/or analyzed during the current study are available from the corresponding author on reasonable request.

\section{Declarations}

Conflict of interest The authors declare no competing interests.

Open Access This article is licensed under a Creative Commons Attribution 4.0 International License, which permits use, sharing, adaptation, distribution and reproduction in any medium or format, as long as you give appropriate credit to the original author(s) and the source, provide a link to the Creative Commons licence, and indicate if changes were made. The images or other third party material in this article are included in the article's Creative Commons licence, unless indicated otherwise in a credit line to the material. If material is not included in the article's Creative Commons licence and your intended use is not permitted by statutory regulation or exceeds the permitted use, you will need to obtain permission directly from the copyright holder. To view a copy of this licence, visit http://creativecommons.org/licenses/by/4.0/.

\section{References}

1. Bandara, H. M. D., \& Burdette, S. C. (2012). Photoisomerization in different classes of azobenzene. Chemical Society Reviews, 41, 1809-1825. https://doi.org/10.1039/c1cs15179g.

2. Irie, M., Fukaminato, T., Matsuda, K., \& Kobatake, S. (2014). Photochromism of diarylethene molecules and crystals: memories, switches, and actuators. Chemical Reviews, 114, 12174-12277. https://doi.org/10.1021/cr500249p.
3. Zhang, J., \& Tian, H. (2018). The endeavor of diarylethenes: new structures, high performance, and bright future. Advanced Optical Materials, 6, 1701278. https://doi.org/10.1002/adom.201701278.

4. Kortekaas, L., \& Browne, W. R. (2019). The evolution of spiropyran: fundamentals and progress of an extraordinarily versatile photochrome. Chemical Society Reviews, 48, 3406-3424. https:// doi.org/10.1039/C9CS00203K.

5. Klajn, R. (2014). Spiropyran-based dynamic materials. Chemical Society Reviews, 43, 148-184. https://doi.org/10.1039/C3CS6 0181A.

6. Lukyanov, B. S., \& Lukyanova, M. B. (2005). Spiropyrans: snthesis, properties, and application. Chemistry of Heterocyclic Compounds, 41, 1-31.

7. Velema, W. A., Szymanski, W., \& Feringa, B. L. (2014). Photopharmacology: beyond proof of principle. Journal of the American Chemical Society, 136, 2178-2191. https://doi.org/10.1021/ja413 063e.

8. Broichhagen, J., Frank, J. A., \& Trauner, D. (2015). A roadmap to success in photopharmacology. Accounts of Chemical Research, 48, 1947-1960. https://doi.org/10.1021/acs.accounts.5b00129.

9. Lerch, M. M., Hansen, M. J., van Dam, G. M., et al. (2016). Emerging targets in photopharmacology. Angewandte Chemie International Edition, 55, 10978-10999. https://doi.org/10. 1002/anie.201601931.

10. Hüll, K., Morstein, J., \& Trauner, D. (2018). In vivo photopharmacology. Chemical Reviews, 118, 10710-10747. https://doi. org/10.1021/acs.chemrev.8b00037.

11. Fuchter, M. J. (2020). On the promise of photopharmacology using photoswitches: a medicinal chemist's perspective. Journal of Medicinal Chemistry, 63, 11436-11447. https://doi.org/10. 1021/acs.jmedchem.0c00629.

12. Neilson, B. M., \& Bielawski, C. W. (2013). Illuminating photoswitchable catalysis. ACS Catalysis, 3, 1874-1885. https://doi. org/10.1021/cs4003673.

13. Göstl, R., Senf, A., \& Hecht, S. (2014). Remote-controlling chemical reactions by light: towards chemistry with high spatiotemporal resolution. Chemical Society Reviews, 43, 1982-1996. https://doi.org/10.1039/c3cs60383k.

14. Stoll, R. S., \& Hecht, S. (2010). Artificial light-gated catalyst systems. Angewandte Chemie -International Edition, 49, 50545075. https://doi.org/10.1002/anie.201000146.

15. Zhang, J., Zou, Q., \& Tian, H. (2013). Photochromic materials: more than meets the eye. Advanced Materials, 25, 378-399. https://doi.org/10.1002/adma.201201521.

16. Goulet-Hanssens, A., Eisenreich, F., \& Hecht, S. (2020). Enlightening materials with photoswitches. Advanced Materials, 32, 1905966. https://doi.org/10.1002/adma.201905966.

17. Moulin, E., Faour, L., Carmona-Vargas, C. C., \& Giuseppone, N. (2020). From molecular machines to stimuli-responsive materials. Advanced Materials, 32, 1906036. https://doi.org/ 10.1002/adma.201906036.

18. Lancia, F., Ryabchun, A., \& Katsonis, N. (2019). Life-like motion driven by artificial molecular machines. Nature Reviews Chemistry, 3, 536-551. https://doi.org/10.1038/ s41570-019-0122-2.

19. Dong, M., Babalhavaeji, A., Samanta, S., et al. (2015). Redshifting Azobenzene Photoswitches for in vivo use. Accounts of Chemical Research, 48, 2662-2670. https://doi.org/10.1021/acs. accounts.5b00270.

20. Mosciatti, T., Bonacchi, S., Gobbi, M., et al. (2016). Optical input/ electrical output memory elements based on a liquid crystalline azobenzene polymer. ACS Applied Materials \& Interfaces, 8, 6563-6569. https://doi.org/10.1021/acsami.5b12430.

21. Shi, Y., Gerkman, M. A., Qiu, Q., et al. (2021). Sunlight-activated phase change materials for controlled heat storage and triggered 
release. Journal of Materials Chemistry A, 9, 9798-9808. https:// doi.org/10.1039/D1TA01007G.

22. Gerkman, M. A., Gibson, R. S. L., Calbo, J., et al. (2020). Arylazopyrazoles for long-term thermal energy storage and optically triggered heat release below $0{ }^{\circ} \mathrm{C}$. Journal of the American Chemical Society, 142, 8688-8695. https://doi.org/10.1021/jacs.0c003 74.

23. Lahikainen, M., Zeng, H., \& Priimagi, A. (2018). Reconfigurable photoactuator through synergistic use of photochemical and photothermal effects. Nature Communications, 9, 4148. https:// doi.org/10.1038/s41467-018-06647-7.

24. Iamsaard, S., Anger, E., Aßhoff, S. J., et al. (2016). Fluorinated azobenzenes for shape-persistent liquid crystal polymer networks. Angewandte Chemie-International Edition, 55, 9908-9912. https://doi.org/10.1002/anie.201603579.

25. Ryabchun, A., Li, Q., Lancia, F., et al. (2019). Shape-persistent actuators from hydrazone photoswitches. Journal of the American Chemical Society, 141, 1196-1200. https://doi.org/10.1021/jacs. $8 \mathrm{~b} 11558$.

26. Lauer, A., Fast, D. E., Steinkoenig, J., et al. (2017). Wavelengthdependent photochemical stability of photoinitiator-derived macromolecular Chain Termini. ACS Macro Letters, 6, 952-958. https://doi.org/10.1021/acsmacrolett.7b00499.

27. Yousif, E., \& Haddad, R. (2013). Photodegradation and photostabilization of polymers, especially polystyrene: Review. Springerplus, 2, 398. https://doi.org/10.1186/2193-1801-2-398.

28. Aleotti, F., Nenov, A., Salvigni, L., et al. (2020). Spectral tuning and photoisomerization efficiency in push-pull azobenzenes: designing principles. Journal of Physical Chemistry A, 124, 9513-9523. https://doi.org/10.1021/acs.jpca.0c08672.

29. Eom, T., \& Khan, A. (2021). Push-pull azobenzene chromophores with negative halochromism. Dyes and Pigments, 188, 109197. https://doi.org/10.1016/j.dyepig.2021.109197.

30. Dokić, J., Gothe, M., Wirth, J., et al. (2009). Quantum chemical investigation of thermal Cis-to-Trans isomerization of azobenzene derivatives: Substituent effects, solvent effects, and comparison to experimental data. Journal of Physical Chemistry A, 113, 6763-6773. https://doi.org/10.1021/jp9021344.

31. Knie, C., Utecht, M., Zhao, F., et al. (2014). Ortho-fluoroazobenzenes: Visible light switches with very long-lived $\mathrm{Z}$ isomers. Chemistry-A. European Journal, 20, 16492-16501. https://doi.org/10.1002/chem.201404649.

32. Siewertsen, R., Neumann, H., Buchheim-Stehn, B., et al. (2009). Highly efficient reversible Z-E photoisomerization of a bridged azobenzene with visible light through resolved $\mathrm{S}(1)(\mathrm{n} \mathrm{pi*})$ absorption bands. Journal of the American Chemical Society, 131, 15594-15595. https://doi.org/10.1021/ja906547d.

33. Beharry, A. A., Sadovski, O., \& Woolley, G. A. (2011). Azobenzene photoswitching without ultraviolet light. Journal of the American Chemical Society, 133, 19684-19687. https://doi.org/ $10.1021 / \mathrm{ja} 209239 \mathrm{~m}$

34. Bléger, D., Schwarz, J., Brouwer, A. M., \& Hecht, S. (2012). o-Fluoroazobenzenes as readily synthesized photoswitches offering nearly quantitative two-way isomerization with visible Light. Journal of the American Chemical Society, 134, 20597-20600. https://doi.org/10.1021/ja310323y.

35. Ditter, D., Braun, L. B., \& Zentel, R. (2020). Influences of ortho-fluoroazobenzenes on liquid crystalline phase stability and 2D (Planar) actuation properties of liquid crystalline elastomers. Macromolecular Chemistry and Physics, 221, 1900265. https://doi.org/10.1002/macp.201900265.

36. Leistner, A.-L., Kirchner, S., Karcher, J., et al. (2021). Fluorinated azobenzenes switchable with red light. Chemistry-A. European Journal, 27, 8094-8099. https://doi.org/10.1002/ chem.202005486.
37. Wegener, M., Hansen, M. J., Driessen, A. J. M., et al. (2017). Photocontrol of antibacterial activity: shifting from UV to Red Light Activation. Journal of the American Chemical Society, 139, 17979-17986. https://doi.org/10.1021/jacs.7b09281.

38. Konrad, D. B., Savasci, G., Allmendinger, L., et al. (2020). Computational design and synthesis of a deeply red-Shifted and bistable azobenzene. Journal of the American Chemical Society, 142, 6538-6547. https://doi.org/10.1021/jacs.9b10430.

39. Lameijer, L. N., Budzak, S., Simeth, N. A., et al. (2020). General principles for the design of visible-light-responsive photoswitches: tetra- ortho -chloro-azobenzenes. Angewandte Chemie. https://doi.org/10.1002/ange.202008700.

40. Sadovski, O., Beharry, A. A., Zhang, F., \& Woolley, G. A. (2009). Spectral tuning of azobenzene photoswitches for biological applications. Angewandte Chemie International Edition, 48, 1484-1486. https://doi.org/10.1002/anie.200805013.

41. Boulègue, C., Löweneck, M., Renner, C., \& Moroder, L. (2007). Redox potential of azobenzene as an amino acid residue in peptides. ChemBioChem, 8, 591-594. https://doi.org/10.1002/cbic. 200600495.

42. Ahmed, Z., Siiskonen, A., Virkki, M., \& Priimagi, A. (2017). Controlling azobenzene photoswitching through combined: Ortho -fluorination and -amination. Chemical Communications, 53, 12520-12523. https://doi.org/10.1039/c7cc07308a.

43. Becke, A. D. (1993). Density-functional thermochemistry. III. The role of exact exchange. The Journal of Chemical Physics, 98, 5648-5652. https://doi.org/10.1063/1.464913.

44. Stephens, P. J., Devlin, F. J., Chabalowski, C. F., \& Frisch, M. J. (1994). Ab initio calculation of vibrational absorption and circular dichroism spectra using density functional force fields. Journal of Physical Chemistry, 98, 11623-11627. https://doi.org/10.1021/ j100096a001.

45. Grimme, S., Ehrlich, S., \& Goerigk, L. (2011). Effect of the damping function in dispersion corrected density functional theory. Journal of Computational Chemistry, 32, 1456-1465. https://doi. org/10.1002/jcc.21759.

46. Hariharan, P. C., \& Pople, J. A. (1973). The influence of polarization functions on molecular orbital hydrogenation energies. Theoretica Chimica Acta, 28, 213-222. https://doi.org/10.1007/BF005 33485.

47. Miertuš, S., Scrocco, E., \& Tomasi, J. (1981). Electrostatic interaction of a solute with a continuum. A direct utilizaion of $\mathrm{AB}$ initio molecular potentials for the prevision of solvent effects. Chemical Physics, 55, 117-129. https://doi.org/10.1016/03010104(81)85090-2.

48. Scalmani, G., \& Frisch, M. J. (2010). Continuous surface charge polarizable continuum models of solvation I. General formalism. The Journal of Chemical Physics, 132, 114110. https://doi.org/ 10.1063/1.3359469.

49. Cusati, T., Granucci, G., Persico, M., \& Spighi, G. (2008). Oscillator strength and polarization of the forbidden $n \rightarrow \pi^{*}$ band of trans-azobenzene: a computational study. The Journal of Chemical Physics, 128, 194312. https://doi.org/10.1063/1.2925678.

50. Hepworth, J. D., Mason, D., Hallas, G., \& Marsden, R. (1985). The effects of cyclic terminal groups in 44minoazo-benzene and related Azo dyes: Part 2'f-p\& values of some monoazo dyes derived from IV-phenylpyrrolidine and N-Phenylpiperidine. Dyes and Pigments, 6, 389-396.

51. Brown, H. C., Brewster, J. H., \& Shechter, H. (1954). An interpretation of the chemical behavior of Five- and Six-membered Ring compounds. Journal of the American Chemical Society, 76, 467-474. https://doi.org/10.1021/ja01631a041.

52. Casida, M. E. (1995). Time-dependent density functional response theory for molecules. in Recent advances in density functional methods (pp. 155-192). World Scientific. 
53. Mennucci, B. (2015). Modeling absorption and fluorescence solvatochromism with QM/Classical approaches. International Journal of Quantum Chemistry, 115, 1202-1208. https://doi.org/ 10.1002/qua.24889.

54. Chai, J.-D., \& Head-Gordon, M. (2008). Long-range corrected hybrid density functionals with damped atom-atom dispersion corrections. Physical Chemistry Chemical Physics: PCCP, 10, 6615-6620. https://doi.org/10.1039/B810189B.

55. Titov, E., \& Saalfrank, P. (2016). Exciton splitting of adsorbed and Free 4-Nitroazobenzene dimers: a quantum chemical study. Journal of Physical Chemistry A, 120, 3055-3070. https://doi.org/ 10.1021/acs.jpca.5b10376.

56. Titov, E., Hummert, J., Ikonnikov, E., et al. (2021). Electronic relaxation of aqueous aminoazobenzenes studied by time-resolved photoelectron spectroscopy and surface hopping TDDFT dynamics calculations. Faraday Discussions. https://doi.org/10.1039/ D0FD00111B.

57. Martin, R. L. (2003). Natural transition orbitals. The Journal of Chemical Physics, 118, 4775-4777. https://doi.org/10.1063/1. 1558471.

58. Sršeň, Š, Sita, J., Slavíček, P., et al. (2020). Limits of the nuclear ensemble method for electronic spectra simulations: temperature dependence of the (E)-azobenzene spectrum. Journal of Chemical Theory and Computation, 16, 6428-6438. https://doi.org/10.1021/ acs.jctc.0c00579.

59. Isokuortti, J., Kuntze, K., Virkki, M., et al. (2021). Expanding excitation wavelengths for azobenzene photoswitching into the near-infrared range via endothermic triplet energy transfer. Chemical Science. https://doi.org/10.1039/D1SC01717A.

60. Goulet-Hanssens, A., Utecht, M., Mutruc, D., et al. (2017). Electrocatalytic $\mathrm{Z} \rightarrow \mathrm{E}$ Isomerization of Azobenzenes. Journal of the American Chemical Society, 139, 335-341. https://doi.org/10. 1021/jacs.6b10822.

61. Goulet-Hanssens, A., Rietze, C., Titov, E., et al. (2018). Hole catalysis as a general mechanism for efficient and wavelengthindependent $\mathrm{Z} \rightarrow \mathrm{E}$ azobenzene isomerization. Chem, 4, 17401755. https://doi.org/10.1016/j.chempr.2018.06.002.

62. Hallett-Tapley, G. L., D’Alfonso, C., Pacioni, N. L., et al. (2013). Gold nanoparticle catalysis of the cis-trans isomerization of azobenzene. Chemical Communications, 49, 10073-10075. https://doi.org/10.1039/C3CC41669K.

63. Titov, E., Lysyakova, L., Lomadze, N., et al. (2015). Thermal Cis-to-Trans isomerization of azobenzene-containing molecules enhanced by gold nanoparticles: an experimental and theoretical study. Journal of Physical Chemistry C, 119, 17369-17377. https://doi.org/10.1021/acs.jpcc.5b02473.

64. Eyring, H. (1935). The activated complex and the absolute rate of chemical reactions. Chemical Reviews, 17, 65-77. https://doi.org/ 10.1021/cr60056a006.

65. Rietze, C., Titov, E., Lindner, S., \& Saalfrank, P. (2017). Thermal isomerization of azobenzenes: on the performance of Eyring transition state theory. Journal of Physics: Condensed Matter, 29, 314002. https://doi.org/10.1088/1361-648x/aa75bd.

66. Fukui, K. (1981). The path of chemical reactions-the IRC approach. Accounts of Chemical Research, 14, 363-368. https:// doi.org/10.1021/ar00072a001.
67. Hratchian, H. P., \& Schlegel, H. B. (2005). Chapter 10 Finding minima, transition states, and following reaction pathways on ab initio potential energy surfaces. In C. E. Dykstra, G. Frenking, \& K. S. Kim (Eds.), Scuseria GEBT-T and A of CC (pp. 195-249). Amsterdam: Elsevier.

68. He, Y., Shangguan, Z., Zhang, Z.-Y., et al. (2021). Azobispyrazole family as photoswitches combining (Near-) Quantitative bidirectional isomerization and widely tunable thermal half-lives from hours to years. Angewandte Chemie International Edition. https:// doi.org/10.1002/anie.202103705.

69. Yamaguchi, K., Jensen, F., Dorigo, A., \& Houk, K. N. (1988). A spin correction procedure for unrestricted Hartree-Fock and Møller-Plesset wavefunctions for singlet diradicals and polyradicals. Chemical Physics Letters, 149, 537-542. https://doi.org/10. 1016/0009-2614(88)80378-6.

70. Gagliardi, L., Orlandi, G., Bernardi, F., et al. (2004). A theoretical study of the lowest electronic states of azobenzene: The role of torsion coordinate in the cis-trans photoisomerization. Theoretical Chemistry Accounts, 111, 363-372. https://doi.org/10.1007/ s00214-003-0528-1.

71. Vela, S., Scheidegger, A., Fabregat, R., \& Corminboeuf, C. (2021). Tuning the thermal stability and photoisomerization of azoheteroarenes through Macrocycle Strain. Chemistry-A European Journal, 27, 419-426. https://doi.org/10.1002/chem.20200 3926.

72. Cembran, A., Bernardi, F., Garavelli, M., et al. (2004). On the mechanism of the cis-trans isomerization in the lowest electronic States of Azobenzene: S0, S1, and T1. Journal of the American Chemical Society, 126, 3234-3243. https://doi.org/10.1021/ja038 $327 \mathrm{y}$.

73. Heindl, A. H., \& Wegner, H. A. (2020). Rational design of azothiophenes - substitution effects on the switching properties. Chemistry-A European Journal, 26, 13730-13737. https://doi. org/10.1002/chem.202001148.

74. Frisch, M. J., Trucks, G. W., Schlegel, H. B., Scuseria, G. E., Robb, M. A., Cheeseman, J. R., Scalmani, G., Barone, V., Petersson, G. A., Nakatsuji, H., Li, X., Caricato, M., Marenich, A. V., Bloino, J., Janesko, B. G., Gomperts, R., Mennucci, B., Hratchian, H. P., Ortiz, J. V., Izmaylov, A. F., Sonnenberg, J. L., WilliamsYoung, D., Ding, F., Lipparini, F., Egidi, F., Goings, J., Peng, B., Petrone, A., Henderson, T., Ranasinghe, D., Zakrzewski, V. G., Gao, J., Rega, N., Zheng, G., Liang, W., Hada, M., Ehara, M., Toyota, K., Fukuda, R., Hasegawa, J., Ishida, M., Nakajima, T., Honda, Y., Kitao, O., Nakai, H., Vreven, T., Throssell, K., Montgomery, J. A., Jr., Peralta, J. E., Ogliaro, F., Bearpark, M. J., Heyd, J. J., Brothers, E. N., Kudin, K. N., Staroverov, V. N., Keith, T. A., Kobayashi, R., Normand, J., Raghavachari, K., Rendell, A. P., Burant, J. C., Iyengar, S. S., Tomasi, J., Cossi, M., Millam, J. M., Klene, M., Adamo, C., Cammi, R., Ochterski, J. W., Martin, R. L., Morokuma, K., Farkas, O., Foresman, J. B. \& Fox, D. J. (2016). Gaussian 16, Revision C.01. Wallingford CT: Gaussian Inc. 